\title{
Complete Z-intuitionistic Fuzzy MULTIMOORA Method with AHP and its Application to COVID-19
}

\section{Yijin Zhang ( $\nabla$ zhangyj@cqupt.edu.cn )}

Chongqing University of Posts and Telecommunications

Jie Huang

Chongqing University of Posts and Telecommunications

Zongbing Lin

Panzhihua University

\section{Research Article}

Keywords: Intuitionistic fuzzy set(IFS), Z-number, Complete Z-intuitionistic set(CZIFS), MULTIMOORA, Multicriteria group decision making, Fangcang shelter hospital

Posted Date: July 6th, 2021

DOI: https://doi.org/10.21203/rs.3.rs-678049/v1

License: (c) (1) This work is licensed under a Creative Commons Attribution 4.0 International License. Read Full License 


\title{
Complete Z-intuitionistic Fuzzy MULTIMOORA Method with AHP and its Application to COVID-19
}

\author{
Yijin Zhang ${ }^{\text {齐a }}$, Jie Huang ${ }^{\mathrm{a}}$, Zongbing Lin $^{\mathrm{b}}$ \\ ${ }^{a}$ Key Lab of Intelligent Analysis and Decision on Complex Systems, \\ Key Laboratory of Industrial Internet of Things and Networked Control, \\ Ministry of Education, Chongqing University of Posts and Telecommunications, Chongqing 400065, P.R. \\ China. \\ ${ }^{b}$ School of Mathematics and Computer Science, Panzhihua University, Panzhihua 617000, P.R. China
}

\begin{abstract}
For emergencies, the reliability of information can not be guaranteed. At the same time, due to the lack of information and knowledge, neither the criteria itself nor the credibility can be given a precise evaluation by decision-makers(DMs). Therefore, we combine intuitionistic fuzzy set and Z-number to get a new class of fuzzy set, complete Z-intuitionistic fuzzy set(CZIFS), and its degenerate form, A-type Z-intuitionistic fuzzy set(AZIFS) and B-type Z-intuitionistic fuzzy set(BZIFS). CZIFS can serve as a reliable tool to depict the hesitant degree both on the ambiguity and reliability of uncertain information. In addition, we introduce the score and accuracy functions and distance measure of complete Z-intuitionistic fuzzy number(CZIFN), with which we have considered both reliability information and DMs' preference on it. Then, we improve traditional MULTIMOORA by developing reference point(RP) model to consider both the risk and profile of alternatives and integrating analytic hierarchy process(AHP) in the process of ranking aggregation method to take into account the preference of DMs on three subordinate rankings. Besides, to solve multicriteria group decision making(MCGDM) problem, we develop improved MULTIMOORA method to the environment of CZIFN. Finally, to illustrate the proposed method, we give a numerical example, solving site selecting of Fangcang shelter hospital for COVID-19.
\end{abstract}

Keywords: Intuitionistic fuzzy set(IFS); Z-number; Complete Z-intuitionistic set(CZIFS); MULTIMOORA; Multicriteria group decision making; Fangcang shelter hospital.

\section{Introduction}

The outbreak of COVID-19 has a great impact on human life and social economic development. In the short term, extraordinarily numerous patients need to be isolated and treated, which overburden the medical system. Fangcang shelter hospital refers to the hospital which is rebuilt in fixed place through large venues or built temporarily with minimum time and less costs, and plays a great role in public health emergency protection such as earthquake, disaster, COVID-19, etc.

\footnotetext{
${ }^{\star}$ Yijin Zhang. E-mail: zhangyj@cqupt.edu.cn (Y.J. Zhang)
} 
Site selecting is a prerequisite for Fangcang shelter hospital. How to choose the optimized one or several places to convert into a Fangcang shelter hospital can be treated as a Multicriteria group decision-making(MCGDM) problem. MCGDM problem is universal and essential in the real world, such as recruitment[1], venture capital[2] and so on. For emergencies, efficient and accurate decision-making is even more critical. COVID-19 virus has a high degree of uncertainty for human beings, and the government or other relevant decision-making institutions can not obtain the prepared information. Hence, it becomes more difficult to make decision. For decision-makers(DMs), the credibility of the information is also uncertain in similar situation, in addition to the uncertainty of evaluating the attribute itself. Therefore, experts have researched the representation of information in terms of ambiguity and credibility.

First, to depict the ambiguity of information, Zadeh[3] proposed the concept of fuzzy set(FS). Then, Atanassov[4] introduced intuitionistic fuzzy set(IFS) characterized by membership and nonmembership degree, which can further represent the hesitancy of information. Later on, by relaxing the constraints of membership degree and nonmembership degree of IFS, Yager[5] first raisen Pythagorean fuzzy set(PFS). Torra[6] put forward hesitant fuzzy set(HFS) to consider hesitant information.

Second, as important as the credibility of information is, such information was not well considered by traditional information representation. In 2011, Zadeh[7] developed the concept of Z-number, which describes both the restriction and the reliability of evaluation. Since Zadeh given the concept of Z-number, scholars have studied Z-number and related problems. On the one hand, some contribute to the extension of Z-number. Banerjee et al.[8] proposed $Z^{*}$-number. Xian et al.[9, 10] introduced $\mathcal{Z}$-number and intuitionistic Z-linguistic set(IZLS). Extended by HFS and Z-number, Peng et al.[11] presented hesitant uncertain linguistic Znumber(HULZN). On the other hand, some contribute to the conversion, computation and ranking problems of Z-numbers[12-27].

Through years of studying, scholars made productive research results on Z-number. But, still, some situations can not be expressed by Z-number properly. For example, assume that the expert is assessing whether the local epidemic situation has improved by using data on the number of local infections. The expert first makes an evaluation $(0.6,0.3)$ based on the number of infected people. The evaluation means that the probability of improvement is $60 \%$, the probability of no improvement is $30 \%$, and another $10 \%$ is between improvement and no improvement, which indicates that the expert is uncertain and hesitant. Besides, for reasons such as incomplete virus detection mechanism, the reliability of the data can not be guaranteed. Thus, the expert also gives an evaluation $(0.7,0.1)$ on the credibility of previous evaluation, which means that the expert believes that the credibility of the evaluation is $70 \%$, the unreliability is $10 \%$, and the other $20 \%$ expresses the expert's hesitation in credibility. In such a situation, ambiguity exists in restriction and reliability of information simultaneously. However, the former representation of information can not express this situation adequately.

When we select optimal one from alternatives in the MCGDM problem, the ranking technique is part and parcel. In 2006, Brauers et al.[28] proposed MOORA (Multi-Objective Optimization on the basis of a Ratio Analysis) method combining Ratio System(RS) and Reference Point Approach(RP). Then Brauers et al.[29] came up with MULTIMOORA method extended from MOORA method by adding Full Multiplicative Form(FMF). It can supply the final integrative ranking based on the results of these triple subordinate methods. MULTI- 
MOORA method has significant features, for example, more simplicity, less mathematical calculations, better stability and shorter solving time than other methods, such as TOPSIS[30], TODIM[31], and VIKOR[32]. With such good features, MULTIMOORA method was studied and developed by scholars. By using gray numbers, Datta et al.[33] improved MULTIMOORA method. Souzangarzadeh et al.[34] developed MULTIMOORA combined with numeric logic method for determining a conical segmented aluminum tubes optimum design. Chen et al.[35] introduced an extended MULTIMOORA method for solving the MCGDM problems and so on.

Based on the above discussion and analysis, there still exist some shortcomings and limitations in previous researches:

(a). In some situation, both the restriction and the reliability of evaluation contain hesitant information. However, previous studies on Z-number and its expanded forms do not simultaneously depict the hesitation of the restriction and the reliability of evaluation. Traditional score and accuracy functions and distance measure do not consider the reliability information or the DMs' preference on reliability information.

(b). MULTIMOORA method is not combined with Z-number, which leads MULTIMOORA method not to associate with reliability information well. Then, the RP model of MULTIMOORA method only take into account profile of alternatives, not their risk. Besides, the ranking aggregation tool of traditional MULTIMOORA method, dominance theory, do not consider DMs' preference on the three subordinate rankings.

(c). In an emergency, the traditional information expression and decision-making methods can not adapt to the MCGDM problems, such as site selecting of fangcang shelter hospital.

In order to overcome these shortcomings and limitations, we propose complete Z-intuition-istic fuzzy MULTIMOORA(CZIF-MA) method. The contributions of this paper can be summarized as follows:

(a). For the superiority of both IFS and Z-number on describing information, we integrate IFS and Z-number to propose a new class of fuzzy set, complete Z-intuitionistic fuzzy set(CZIFS), and its degenerate form, A-type Z-intuitionistic fuzzy set(AZIFS) and B-type Z-intuitionistic fuzzy set(BZIFS). In addittion, we introduce the score and accuracy functions and distance measure of complete Z-intuitionistic fuzzy number(CZIFN) which consider both reliability information and DMs' preference on reliability information.

(b). We improve traditional MULTIMOORA method from two aspects. First, the RP model of MULTIMOORA method is improved to take into account both profile and risk of alternatives. Second, Analytic hierarchy process(AHP) is introduced in the process of ranking aggregation of MULTIMOORA method to reflect DMs' preference on three subordinate rankings. Then, we combine improved MULTIMOORA method with CZIFS to obtain CZIF-MA method.

(c). To solve MCGDM problem, we propose an approach for MCGDM based on CZIF-MA 
with AHP, complete Z-intuitionistic fuzzy induced ordered weighted average(CZIIOWA) operator and complete Z-intuitionistic fuzzy induced ordered weighted geometric(CZIIOWG) operator. Location problem of Fangcang shelter hospital for COVID-19 is solved by the proposed method.

The rest of this paper is organized as follows. In Section 2, we briefly review some basic concepts. In Section 3, we introduce CZIFS, and its degenerate forms, AZIFS and BZIFS, and corresponding operation rules. Then, a method for ranking CZIFNs based on score and accuracy functions is proposed. In Section 4, we develop CZIIOWA and CZIIOWG operators. In Section 5, the Z-intuitionistic fuzzy MULTIMOORA method with AHP is investigated and the procedure of the proposed method is described in detail. In Section 6, we give a numerical example, site selecting for Fangcang shelter hospital, to illustrate practicability and reliability of the proposed method. Eventually, the conclusion is conducted in Section 7.

\section{Preliminaries}

In this section, we briefly review some concepts. These basic concepts are used throughout this paper unless stated otherwise.

\subsection{Intuitionistic fuzzy set}

Atanassov[4] put forward the definition of the intuitionistic fuzzy set(IFS). IFS is defined as a set of ordered pairs of membership and nonmembership in a non-empty universe of discourse $X$, which is an extention measure of fuzzy or uncertain information.

Definition 1. [4] Let $X$ be a non-empty universe of discourse. An intuitionistic fuzzy set $A$ in $X$ is defined as:

$$
A=\left\{\left\langle x, \mu_{A}(x), \nu_{A}(x)\right\rangle \mid x \in X\right\},
$$

where $\mu_{A}(x): X \rightarrow[0,1]$ and $\nu_{A}(x): X \rightarrow[0,1]$ represent the membership and nonmembership degree of $x \in X$ to $A$ respectively with the condition: $0 \leq \mu_{A}(x)+\nu_{A}(x) \leq 1$. In addition, the degree of indeterminacy (or the hesitation degree) is defined as follows:

$$
\pi_{A}(x)=1-\mu_{A}(x)-\nu_{A}(x),
$$

which expresses a lack of knowledge regarding whether $x$ belongs to $A$.

Definition 2. [4] Let $\alpha=(\mu, \nu), \alpha_{1}=\left(\mu_{1}, \nu_{1}\right)$ and $\alpha_{2}=\left(\mu_{2}, \nu_{2}\right)$ be any three IFNs. The basic operation rules of IFNs are given as follows:

(1) $\bar{\alpha}=(\nu, \mu)$;

(2) $\alpha_{1} \vee \alpha_{2}=\left(\max \left\{\mu_{1}, \mu_{2}\right\}, \min \left\{\nu_{1}, \nu_{2}\right\}\right)$;

(3) $\alpha_{1} \wedge \alpha_{2}=\left(\min \left\{\mu_{1}, \mu_{2}\right\}, \max \left\{\nu_{1}, \nu_{2}\right\}\right)$;

(4) $\alpha_{1} \oplus \alpha_{2}=\left(\mu_{1}+\mu_{2}-\mu_{1} \mu_{2}, \nu_{1} \nu_{2}\right)$

(5) $\alpha_{1} \otimes \alpha_{2}=\left(\mu_{1} \mu_{2}, \nu_{1}+\nu_{2}-\nu_{1} \nu_{2}\right)$;

(6) $\lambda \alpha=\left(1-(1-\mu)^{\lambda}, \nu^{\lambda}\right), \quad \lambda>0$;

(7) $\alpha^{\lambda}=\left(\mu^{\lambda}, 1-(1-\nu)^{\lambda}\right), \quad \lambda>0$. 
Theorem 2.1. [4] Let $\alpha=(\mu, \nu), \alpha_{1}=\left(\mu_{1}, \nu_{1}\right)$ and $\alpha_{2}=\left(\mu_{2}, \nu_{2}\right)$ be any three IFNs. The operation rules have the following properties:

(1) $\alpha_{1} \oplus \alpha_{2}=\alpha_{2} \oplus \alpha_{1}$;

(2) $\alpha_{1} \otimes \alpha_{2}=\alpha_{2} \otimes \alpha_{1}$

(3) $\lambda\left(\alpha_{1} \oplus \alpha_{2}\right)=\lambda \alpha_{1} \oplus \lambda \alpha_{2}, \quad \lambda>0$;

(4) $\alpha^{\lambda_{1}+\lambda_{2}}=\alpha^{\lambda_{1}} \otimes \alpha^{\lambda_{2}}, \quad \lambda_{1}>0, \lambda_{2}>0$.

\subsection{Z-number}

In the real world, when we describe realistic phenomenon, except ambiguity of the event, the reliability of the event should be considered as it is influential to the authenticity of the information we get. In 2011, Zadeh[7] proposed Z-number, a set of ordered fuzzy numbers, to model both ambiguity and reliability of uncertain information. It can be defined as an ordered pair of fuzzy number as follows.

Definition 3. [7] A Z-number is an ordered pair of fuzzy numbers denoted by $Z=(A, B)$. The first component, $A$, is a restriction (constraint) on the values which is a real-valued uncertain variable. The second, $B$, is a measure of reliability (certainty) of the first component.

\section{Complete Z-intuitionistic fuzzy set}

In this section, we introduce CZIFS combined by IFS and Z-number, and degenerate forms of CZIFS, which are AZIFS and BZIFS. Then, a method for ranking CZIFNs based on score and accuracy functions is proposed.

\subsection{Complete Z-intuitionistic fuzzy set}

Z-number made great progress in the filed of describing uncertain information by adding the measure of reliability of information, but the vagueness and uncertainty in real world still can't be represented in detail by Z-number, composed of FS. The IFS introduced by Atanassov can represent hesitancy and ambiguity of information more comprehensively due to its describing both the membership- and nonmembership- degree. For the superiority of both IFS and Z-number on describing information, we integrate IFS and Z-number to obtain CZIFS. Besides, considering that we may not be able to collect intuitionistic fuzzy evaluation information of both restriction and reliability at the same time, the degenerate forms of CZIFS, which are AZIFS and BZIFS, are proposed in this section. AZIFS and BZIFS represent the hesitation degree in restriction and reliability of information respectively.

Definition 4. Let $X$ be a non-empty universe of discourse. A CZIFS $Z I$ on $X$ is given as:

$$
\begin{aligned}
Z I & =\{\langle x, A I(x), B I(x)\rangle \mid x \in X\} \\
& =\left\{\left\langle x,\left(\mu_{A I}(x), \nu_{A I}(x)\right),\left(\mu_{B I}(x), \nu_{B I}(x)\right)\right\rangle \mid x \in X\right\},
\end{aligned}
$$

where $A I(x)=\left\{\left\langle x, \mu_{A I}(x), \nu_{A I}(x)\right\rangle \mid x \in X\right\}$ and $B I(x)=\left\{\left\langle x, \mu_{B I}(x), \nu_{B I}(x)\right\rangle \mid x \in X\right\}$ are two IFSs, in which $\mu_{A I}(x)$ and $\nu_{A I}(x)$ represent the membership and non-membership degree of $x \in X$ to $Z I$, and $\mu_{B I}(x)$ and $\nu_{B I}(x)$ represent the membership and non-membership degree of reliability of $A I(x)$ respectively. In addition, the degree of indeterminacy (or the hesitation degree) relative to $A I$ and $B I$ are defined as follows:

$$
\pi_{A I}(x)=1-\mu_{A I}(x)-\nu_{A I}(x),
$$




$$
\pi_{B I}(x)=1-\mu_{B I}(x)-\nu_{B I}(x),
$$

which express a lack of knowledge regarding whether $x \in X$ belongs to $Z I$ and whether $A I(x)$ is reliable respectively.

Remark 3.1. Let $X$ be a non-empty universe of discourse. An AZIFS $Z I_{A}$ on $X$ is proposed as:

$$
\begin{aligned}
Z I_{A} & =\{\langle x, A I(x), B(x)\rangle \mid x \in X\} \\
& =\left\{\left\langle x,\left(\mu_{A I}(x), \nu_{A I}(x)\right), \mu_{B}(x)\right\rangle \mid x \in X\right\},
\end{aligned}
$$

where $A I(x)=\left\{\left\langle x, \mu_{A I}(x), \nu_{A I}(x)\right\rangle \mid x \in X\right\}$ is an IFS, in which $\mu_{A I}(x)$ and $\nu_{A I}(x)$ represent the membership and non-membership degree of $x \in X$ to $Z I_{A}$ represently, and $B(x)$, a measure of reliability of $A I(x)$, is a FS, in which $\mu_{B}(x): X \rightarrow[0,1]$. In addition, the degree of indeterminacy (or the hesitation degree) relative to $A I$ is defined as follow:

$$
\pi_{A I}(x)=1-\mu_{A I}(x)-\nu_{A I}(x)
$$

which expresses a lack of knowledge regarding whether $x \in X$ belongs to $Z I_{A}$.

The intuitionistic Z-linguistic set(IZLS) proposed by Xian et al.[10] in 2018 is the linguistic term set form of AZIFS.

Remark 3.2. Let $X$ be a non-empty universe of discourse. A BZIFS $Z I_{B}$ on $X$ is defined as:

$$
\begin{aligned}
Z I_{B} & =\{\langle x, A(x), B I(x)\rangle \mid x \in X\} \\
& =\left\{\left\langle x, \mu_{A}(x),\left(\mu_{B I}(x), \nu_{B I}(x)\right)\right\rangle \mid x \in X\right\},
\end{aligned}
$$

where $A(x)$, a restriction on the values which $x \in X$ to $Z I_{B}$ can take, is a FS in which $\mu_{A}(x): X \rightarrow[0,1]$, and $B I(x)=\left\{\left\langle x, \mu_{B I}(x), \nu_{B I}(x)\right\rangle \mid x \in X\right\}$ is an IFS, in which $\mu_{B I}(x)$ and $\nu_{B I}(x)$ represent the membership and non-membership degree of reliability of $A I(x)$ respectively. In addition, the degree of indeterminacy (or the hesitation degree) relative to $B I$ is defined as follow:

$$
\pi_{B I}(x)=1-\mu_{B I}(x)-\nu_{B I}(x),
$$

which expresses a lack of knowledge regarding whether $A(x)$ is reliable.

For convenience, we represent $Z I=(A I, B I)=\left(\left(\mu_{A I}, \nu_{A I}\right),\left(\mu_{B I}, \nu_{B I}\right)\right), Z I_{A}=(A I, B)=$ $\left(\left(\mu_{A I}, \nu_{A I}\right), \mu_{B}\right)$ and $Z I_{B}=(A, B I)=\left(\mu_{A},\left(\mu_{B I}, \nu_{B I}\right)\right)$ as complete Z-intuitionistic fuzzy number(CZIFN), A-type Z-intuitionistic fuzzy number(AZIFN) and B-type Z-intuitionistic fuzzy number(BZIFN) respectively, where $0 \leq \mu_{A I}, v_{A I}, \mu_{B I}, v_{B I}, \mu_{A}, \mu_{B} \leq 1,0 \leq \mu_{A I}+v_{A I} \leq 1$, and $0 \leq \mu_{B I}+v_{B I} \leq 1$ for each CZIFN, AZIFN or BZIFN.

Example 3.1. Consider the case mentioned in the Introduction. The evaluation proposed by the expert can be expressed as a CZIFN, denoted by $Z I=((0.6,0.3),(0.7,0.1))$. Assuming that experts have a clearer evaluation on whether the local epidemic situation has improved or on the reliability of the information, the evaluation proposed by the expert can be expressed as a AZIFN or BZIFN, denoted by $Z I_{A}=((0.6,0.3), 0.7)$ or $Z I_{B}=(0.6,(0.7,0.1))$. 
Theorem 3.1. Let $Z I$ be a CZIFS. $Z I=\{\langle x, A I(x), B I(x)\rangle \mid x \in X\}=\left\{\left\langle x,\left(\mu_{A I}(x), \nu_{A I}(x)\right)\right.\right.$, $\left.\left.\left(\mu_{B I}(x), \nu_{B I}(x)\right)\right\rangle \mid x \in X\right\}$. We can get the following easily:

(1) If $\nu_{B I}(x)=1-\mu_{B I}(x)$, then $Z I$ reduces to an AZIFS;

(2) If $\nu_{A I}(x)=1-\mu_{A I}(x)$, then $Z I$ reduces to a BZIFS;

(3) If $\nu_{A I}(x)=1-\mu_{A I}(x)$ and $\nu_{B I}(x)=1-\mu_{B I}(x)$, then $Z I$ reduces to a Z-number set;

(4) If $\mu_{B I}(x)=1$, then $Z I$ reduces to an IFS.

\subsection{Computation with CZIFNs}

Since Zadeh proposed Z-number, for application of Z-number, it is essential how to compute with Z-number. Inspired by Aliev et al.[36], the rules of computation with CZIFNs are introduced as following:

Definition 5. Let $Z I=(A I, B I)=\left(\left(\mu_{A I}, \nu_{A I}\right),\left(\mu_{B I}, \nu_{B I}\right)\right), Z I_{1}=\left(A I_{1}, B I_{1}\right)=\left(\left(\mu_{A I_{1}}, \nu_{A I_{1}}\right)\right.$, $\left.\left(\mu_{B I_{1}}, \nu_{B I_{1}}\right)\right)$ and $Z I_{2}=\left(A I_{2}, B I_{2}\right)=\left(\left(\mu_{A I_{2}}, \nu_{A I_{2}}\right),\left(\mu_{B I_{2}}, \nu_{B I_{2}}\right)\right)$ be any three CZIFNs. The operation rules are given as following:

(1) $Z I_{1} \oplus Z I_{2}$

$=\left(A I_{1} \oplus A I_{2}, B I_{1} \oplus B I_{2}\right)$

$=\left(\left(\mu_{A I_{1}}+\mu_{A I_{2}}-\mu_{A I_{1}} \mu_{A I_{2}}, \nu_{A I_{1}} \nu_{A I_{2}}\right),\left(\mu_{B I_{1}}+\mu_{B I_{2}}-\mu_{B I_{1}} \mu_{B I_{2}}, \nu_{B I_{1}} \nu_{B I_{2}}\right)\right)$;

(2) $Z I_{1} \otimes Z I_{2}$

$=\left(A I_{1} \otimes A I_{2}, B I_{1} \otimes B I_{2}\right)$

$=\left(\left(\mu_{A I_{1}} \mu_{A I_{2}}, \nu_{A I_{1}}+\nu_{A I_{2}}-\nu_{A I_{1}} \nu_{A I_{2}}\right),\left(\mu_{B I_{1}} \mu_{B I_{2}}, \nu_{B I_{1}}+\nu_{B I_{2}}-\nu_{B I_{1}} \nu_{B I_{2}}\right)\right)$;

(3) $\lambda Z I=(\lambda A I, \lambda B I)=\left(\left(1-\left(1-\mu_{A I}\right)^{\lambda}, \nu_{A I}^{\lambda}\right),\left(1-\left(1-\mu_{B I}\right)^{\lambda}, \nu_{B I}^{\lambda}\right)\right), \quad \lambda>0$;

(4) $Z I^{\lambda}=\left(A I^{\lambda}, B I^{\lambda}\right)=\left(\left(\mu_{A I}^{\lambda}, 1-\left(1-\nu_{A I}\right)^{\lambda}\right),\left(\mu_{B I}^{\lambda}, 1-\left(1-\nu_{B I}\right)^{\lambda}\right)\right), \quad \lambda>0$.

Example 3.2. Suppose that $Z I_{1}=((0.7,0.2),(0.3,0.5))$ and $Z I_{2}=((0.6,0.1),(0.7,0.1))$ are two CZIFNs and $\lambda=2$. Then:

(1) $Z I_{1} \oplus Z I_{2}$

$=((0.7+0.6-0.7 \times 0.6,0.2 \times 0.1),(0.3+0.7-0.3 \times 0.7,0.5 \times 0.1))$

$=((0.88,0.02),(0.79,0.05))$;

(2) $Z I_{1} \otimes Z I_{2}$

$=((0.7 \times 0.6,0.2+0.1-0.2 \times 0.1),(0.3 \times 0.7,0.5+0.1-0.5 \times 0.1))$

$=((0.42,0.28),(0.21,0.55))$

(3) $\lambda Z I_{1}=\left(\left(1-(1-0.7)^{2}, 0.2^{2}\right),\left(1-(1-0.3)^{2}, 0.5^{2}\right)\right)=((0.91,0.04),(0.51,0.25))$;

(4) $Z I_{1}{ }^{\lambda}=\left(\left(0.7^{2}, 1-(1-0.2)^{2}\right),\left(0.3^{2}, 1-(1-0.5)^{2}\right)\right)=((0.49,0.36),(0.09,0.75))$.

Theorem 3.2. For any two CZIFNs $Z I_{1}=\left(A I_{1}, B I_{1}\right)=\left(\left(\mu_{A I_{1}}, \nu_{A I_{1}}\right),\left(\mu_{B I_{1}}, \nu_{B I_{1}}\right)\right)$ and $Z I_{2}=\left(A I_{2}, B I_{2}\right)=\left(\left(\mu_{A I_{2}}, \nu_{A I_{2}}\right),\left(\mu_{B I_{2}}, \nu_{B I_{2}}\right)\right)$. The operation rules have the following properties:

(1) $Z I_{1} \oplus Z I_{2}=Z I_{2} \oplus Z I_{1}$;

(2) $Z I_{1} \otimes Z I_{2}=Z I_{2} \otimes Z I_{1}$

(3) $\lambda\left(Z I_{1} \oplus Z I_{2}\right)=\lambda Z I_{1} \oplus \lambda Z I_{2}, \quad \lambda>0$;

(4) $Z I_{1}^{\lambda_{1}+\lambda_{2}}=Z I_{1}^{\lambda_{1}} \otimes Z I_{1}^{\lambda_{2}}, \quad \lambda_{1}>0, \lambda_{2}>0$.

\subsection{A method for ranking CZIFNs}

The score and accuracy functions have been used as a common method for ranking IFNs. Therefore, inspired by Liu et al.[37], we proposed the score and accuracy functions of CZIFNs to rank CZIFNs. 
Definition 6. Suppose $Z I=(A I, B I)=\left(\left(\mu_{A I}, \nu_{A I}\right),\left(\mu_{B I}, \nu_{B I}\right)\right)$ is a CZIFN. The score function $S(Z I)$ is given as following:

$$
S(Z I)=p_{Z I}^{k}\left(\mu_{A I}+\left(1-\nu_{A I}\right)\right),
$$

where $k \geq 0$ and $p_{Z I}=\frac{\mu_{B I}}{\mu_{B I}+v_{B I}} \cdot p_{Z I}$ is a standard reliability measure of $Z I$.

Definition 7. Suppose $Z I=(A I, B I)=\left(\left(\mu_{A I}, \nu_{A I}\right),\left(\mu_{B I}, \nu_{B I}\right)\right)$ is a CZIFN. The accuracy function $H(Z I)$ is given as following:

$$
H(Z I)=p_{Z I}^{k}\left(1-\mu_{A I}-\nu_{A I}\right),
$$

where $k \geq 0$ and $p_{Z I}=\frac{\mu_{B I}}{\mu_{B I}+v_{B I}} \cdot p_{Z I}$ is a standard reliability measure of $Z I$.

We introduce the variable $k$ into the score and accuracy functions to control the impact which results from reliability information on decision-making. Theorem 3.3-3.6 show that how the variable $k$ affects the influence degree of credibility in score and accuracy functions.

Theorem 3.3. When $k=0$, it means that reliability information in score and accuracy functions has no effect.

Proof. In Eq.(10) and Eq.(11), when $k=0$, then $p_{Z I}$, as a standard reliability measure of $Z I$, is identically equal to 1 , which indicates that the values of $S(Z I)$ and $H(Z I)$ are independent of reliability.

Theorem 3.4. When $0<k<1$, it means that the effect of reliability information in score and accuracy functions is strong.

Proof. In Eq.(10) and Eq.(11), when $0<k<1$, then $p_{Z I}{ }^{k} \geq p_{Z I}$, which indicates that reliability information has strong effect on the values of $S(Z I)$ and $H(Z I)$.

Theorem 3.5. When $k=1$, it means that the effect of reliability information in score and accuracy functions is neutral.

Proof. In Eq.(10) and Eq.(11), When $k=1$, then $p_{Z I}{ }^{k}=p_{Z I}$, which indicates that the impact, reliability information made on the values of $S(Z I)$ and $H(Z I)$, only depends on $p_{Z I}$ itself, and it is neutral.

Theorem 3.6. When $k>1$, it means that the effect of reliability information in score and accuracy functions is weak.

Proof. In Eq.(10) and Eq.(11), when $k>1$, then $p_{Z I}{ }^{k} \leq p_{Z I}$, which indicates that reliability information has weak effect on the values of $S(Z I)$ and $H(Z I)$.

Let $Z I_{1}=\left(A I_{1}, B I_{1}\right)=\left(\left(\mu_{A I_{1}}, \nu_{A I_{1}}\right),\left(\mu_{B I_{1}}, \nu_{B I_{1}}\right)\right)$ and $Z I_{2}=\left(A I_{2}, B I_{2}\right)=\left(\left(\mu_{A I_{2}}, \nu_{A I_{2}}\right)\right.$, $\left.\left(\mu_{B I_{2}}, \nu_{B I_{2}}\right)\right)$ be any two CZIFNs. Then: 
(1) If $S\left(Z I_{1}\right)>S\left(Z I_{2}\right)$, then $Z I_{1}>Z I_{2}$;

(2) If $S\left(Z I_{1}\right)<S\left(Z I_{2}\right)$, then $Z I_{1}<Z I_{2}$;

(3) If $S\left(Z I_{1}\right)=S\left(Z I_{2}\right)$, then :

If $H\left(Z I_{1}\right)>H\left(Z I_{2}\right)$, then $Z I_{1}>Z I_{2}$;

If $H\left(Z I_{1}\right)<H\left(Z I_{2}\right)$, then $Z I_{1}<Z I_{2}$;

If $H\left(Z I_{1}\right)=H\left(Z I_{2}\right)$, then $Z I_{1}=Z I_{2}$.

\section{CZIIOWA operator and CZIIOWG operator}

Since IOWA[38] and IOWG[39] operators were proposed, they have been widely used to solve MCGDM problems. However, IOWA and IOWG operators are based on traditional information representation, and such a way dose not take into account reliability information. Now, we introduce CZIIOWA and CZIIOWG operators, which consider reliability information.

Definition 8. Let $Z I_{i}=\left(A I_{i}, B I_{i}\right)=\left(\left(\mu_{A I_{i}}, \nu_{A I_{i}}\right),\left(\mu_{B I_{i}}, \nu_{B I_{i}}\right)\right) \quad(i=1,2, \cdots, n)$ be a series of CZIFNs. A CZIIOWA operator of dimention $n$ is a mapping CZIIOWA: $(R \times Z I)^{n} \rightarrow$ $Z I$, to which a weight vector $\omega=\left(\omega_{1}, \omega_{2}, \cdots, \omega_{n}\right)^{T}$ is associated to the set of second arguments of a list of $n$ pairs $\left(u_{i}, Z I_{i}\right) \quad(i=1,2,3, \cdots, n)$ with the conditions $\omega_{i} \in[0,1]$ and $\sum_{i=1}^{n} \omega_{i}=1$. CZIIOWA is subject to the following expression:

$$
\begin{array}{r}
C Z I I O W A_{\omega}\left(\left(u_{1}, Z I_{1}\right),\left(u_{2}, Z I_{2}\right), \cdots,\left(u_{n}, Z I_{n}\right)\right) \\
=\omega_{1} Z I_{\sigma(1)} \oplus \omega_{2} Z I_{\sigma(2)} \oplus \cdots \oplus \omega_{n} Z I_{\sigma(n)},
\end{array}
$$

where $\sigma:(1,2, \cdots, n) \rightarrow(1,2, \cdots, n)$ is a permutation such that $u_{\sigma(1)} \geq u_{\sigma(2)} \geq \cdots \geq$ $u_{\sigma(n-1)} \geq u_{\sigma(n)}$, that is, $\left(u_{\sigma(i)}, Z I_{\sigma(i)}\right)$ is the pair in which $u_{\sigma(i)}$ is the $i$ th largest value in the set $\left\{u_{1}, u_{2}, \cdots, u_{n}\right\} . Z I_{1}, Z I_{2}, \cdots, Z I_{n}$ are induced by the ordering of the values $u_{1}, u_{2}, \cdots, u_{n}$. $u_{1}, u_{2}, \cdots, u_{n}$ are defined as the values of order-inducing variable and $Z I_{1}, Z I_{2}, \cdots, Z I_{n}$ as the values of the argument variable.

Theorem 4.1. Let $\left(u_{n}, Z I_{n}\right) \quad(i=1,2,3, \cdots, n)$ be a series of CZIIOWA pairs. $Z I_{i}$ in $\left(u_{i}, Z I_{i}\right)$ is a CZIFN, denoted by $Z I_{i}=\left(A I_{i}, B I_{i}\right)=\left(\left(\mu_{A I_{i}}, \nu_{A I_{i}}\right),\left(\mu_{B I_{i}}, \nu_{B I_{i}}\right)\right)$. Then, their aggregated value by using the CZIIOWA operator is also a CZIFN, and

$$
\begin{aligned}
C Z I I O W & A_{\omega}\left(\left(u_{1}, Z I_{1}\right),\left(u_{2}, Z I_{2}\right), \cdots,\left(u_{n}, Z I_{n}\right)\right) \\
& =\omega_{1} Z I_{\sigma(1)} \oplus \omega_{2} Z I_{\sigma(2)} \oplus \cdots \oplus \omega_{n} Z I_{\sigma(n)} \\
& =\left(\left(1-\prod_{i=1}^{n}\left(1-\mu_{A I_{\sigma(i)}}\right), \prod_{i=1}^{\omega_{i}} v_{A I_{\sigma(i)}}^{\omega_{i}}\right),\left(1-\prod_{i=1}^{n}\left(1-\mu_{B I_{\sigma(i)}}\right), \prod_{i=1}^{\omega_{i}} v_{B I_{\sigma(i)}}^{\omega_{i}}\right)\right),
\end{aligned}
$$

where $\sigma:(1,2, \cdots, n) \rightarrow(1,2, \cdots, n)$ is a permutation, and $\left(u_{\sigma(i)}, Z I_{\sigma(i)}\right)$ is the pair in which $u_{\sigma(i)}$ is the $i$ th largest value in the set $\left\{u_{1}, u_{2}, \cdots, u_{n}\right\} . Z I_{1}, Z I_{2}, \cdots, Z I_{n}$ are induced by the ordering of the values $u_{1}, u_{2}, \cdots, u_{n} . \omega=\left(\omega_{1}, \omega_{2}, \cdots, \omega_{n}\right)^{T}$ is a weight vector associated to 
the set of second arguments of a list of $n$ pairs $\left(u_{i}, Z I_{i}\right)(i=1,2,3, \cdots, n)$ with the conditions $\omega_{i} \in[0,1]$ and $\sum_{i=1}^{n} \omega_{i}=1$.

Proof. For $n=2$, since

$$
\begin{aligned}
& Z I_{\sigma(1)}=\left(A I_{\sigma(1)}, B I_{\sigma(1)}\right)=\left(\left(\mu_{A I_{\sigma(1)}}, \nu_{A I_{\sigma(1)}}\right),\left(\mu_{B I_{\sigma(1)}}, \nu_{B I_{\sigma(1)}}\right)\right), \\
& Z I_{\sigma(2)}=\left(A I_{\sigma(2)}, B I_{\sigma(2)}\right)=\left(\left(\mu_{A I_{\sigma(2)}}, \nu_{A I_{\sigma(2)}}\right),\left(\mu_{B I_{\sigma(2)}}, \nu_{B I_{\sigma(2)}}\right)\right),
\end{aligned}
$$

we obtain

$$
\begin{aligned}
& \text { CZIIOW } A_{\omega}\left(\left(u_{1}, Z I_{1}\right),\left(u_{2}, Z I_{2}\right)\right) \\
& =\omega_{1} Z I_{\sigma(1)} \oplus \omega_{2} Z I_{\sigma(2)} \\
& =\left(\begin{array}{c}
\left(1-\left(1-\mu_{A I_{\sigma(1)}}\right)^{\omega_{1}}\left(1-\mu_{A I_{\sigma(2)}}\right)^{\omega_{2}}, v_{A I_{\sigma(1)}}^{\omega_{1}} v_{A I_{\sigma(2)}}^{\omega_{2}}\right), \\
\left(1-\left(1-\mu_{B I_{\sigma(1)}}\right)^{\omega_{1}}\left(1-\mu_{B I_{\sigma(2)}}\right)^{\omega_{2}}, v_{B I_{\sigma(1)}}^{\omega_{1}} v_{B I_{\sigma(2)}}^{\omega_{2}}\right.
\end{array}\right),
\end{aligned}
$$

so, Eq.(13) holds.

Suppose that Eq.(13) holds for $n=k, k \in N$, that is

$$
\begin{aligned}
& \text { CZIIOW } A_{\omega}\left(\left(u_{1}, Z I_{1}\right),\left(u_{2}, Z I_{2}\right), \cdots,\left(u_{k}, Z I_{k}\right)\right) \\
& \quad=\omega_{1} Z I_{\sigma(1)} \oplus \omega_{2} Z I_{\sigma(2)} \oplus \cdots \oplus \omega_{k} Z I_{\sigma(k)} \\
& \quad=\left(\left(1-\prod_{i=1}^{k}\left(1-\mu_{A I_{\sigma(i)}}\right)^{\omega_{i}}, \prod_{i=1}^{k} v_{A I_{\sigma(i)}}^{\omega_{i}}\right),\left(1-\prod_{i=1}^{k}\left(1-\mu_{B I_{\sigma(i)}}\right)^{\omega_{i}}, \prod_{i=1}^{k} v_{B I_{\sigma(i)}}^{\omega_{i}}\right)\right),
\end{aligned}
$$

Then, when $n=k+1$, using the computation laws in Def. (5), we have

$$
\begin{aligned}
& C Z I I O W A_{\omega}\left(\left(u_{1}, Z I_{1}\right),\left(u_{2}, Z I_{2}\right), \cdots,\left(u_{k+1}, Z I_{k+1}\right)\right) \\
& =\omega_{1} Z I_{\sigma(1)} \oplus \omega_{2} Z I_{\sigma(2)} \oplus \cdots \oplus \omega_{k} Z I_{\sigma(k+1)} \\
& =\left(\begin{array}{l}
\left(\begin{array}{c}
1-\prod_{i=1}^{k}\left(1-\mu_{A I_{\sigma(i)}}\right)^{\omega_{i}}+1-\left(1-\mu_{A I_{\sigma(k+1)}}\right)^{\omega_{k+1}} \\
-\left(1-\prod_{i=1}^{k}\left(1-\mu_{A I_{\sigma(i)}}\right)^{\omega_{i}}\right)\left(1-\left(1-\mu_{A I_{\sigma(k+1)}}\right)^{\omega_{k+1}}\right)
\end{array}\right), v_{A I_{\sigma(k+1)}^{\omega_{k+1}} \prod_{i=1}^{k} v_{A I_{\sigma(i)}}^{\omega_{i}}}
\end{array}\right),
\end{aligned}
$$




$$
=\left(\begin{array}{c}
\left(1-\prod_{i=1}^{k+1}\left(1-\mu_{A I_{\sigma(i)}}\right), \prod_{i=1}^{\omega_{i}} v_{A I_{\sigma(i)}}^{\omega_{i}}\right) \\
\left(1-\prod_{i=1}^{k+1}\left(1-\mu_{B I_{\sigma(i)}}\right), \prod_{i=1}^{\omega_{i}} v_{B I_{\sigma(i)}}^{\omega_{i}}\right.
\end{array}\right),
$$

thus, Eq.(13) holds for all $n \in N$.

Theorem 4.2. (Commutativity) Let $\left(\left(u_{1}{ }^{*}, Z I_{1}{ }^{*}\right),\left(u_{2}{ }^{*}, Z I_{2}{ }^{*}\right), \cdots,\left(u_{n}{ }^{*}, Z I_{n}{ }^{*}\right)\right)$ be any permutation of the CZIFN vector $\left(\left(u_{1}, Z I_{1}\right),\left(u_{2}, Z I_{2}\right), \cdots,\left(u_{n}, Z I_{n}\right)\right)$, then

$$
\begin{aligned}
C Z I I O W A_{\omega}\left(\left(u_{1}, Z I_{1}\right),\left(u_{2}, Z I_{2}\right), \cdots,\left(u_{n}, Z I_{n}\right)\right) \\
=C Z I I O W A_{\omega}\left(\left(u_{1}^{*}, Z I_{1}^{*}\right),\left(u_{2}{ }^{*}, Z I_{2}{ }^{*}\right), \cdots,\left(u_{n}^{*}, Z I_{n}{ }^{*}\right)\right) .
\end{aligned}
$$

Proof. According to Eq.(12),

$C Z I I O W A_{\omega}\left(\left(u_{1}, Z I_{1}\right),\left(u_{2}, Z I_{2}\right), \cdots,\left(u_{n}, Z I_{n}\right)\right)=\omega_{1} Z I_{\sigma(1)} \oplus \omega_{2} Z I_{\sigma(2)} \oplus \cdots \oplus \omega_{k} Z I_{\sigma(n)}$, $C Z I I O W A_{\omega}\left(\left(u_{1}{ }^{*}, Z I_{1}{ }^{*}\right),\left(u_{2}{ }^{*}, Z I_{2}{ }^{*}\right), \cdots,\left(u_{n}{ }^{*}, Z I_{n}{ }^{*}\right)\right)=\omega_{1} Z I_{\sigma(1)}^{*} \oplus \omega_{2} Z I_{\sigma(2)}^{*} \oplus \cdots \oplus \omega_{k} Z I_{\sigma(n)}^{*}$.

We have $Z I_{\sigma(i)}=Z I_{\sigma(i)}^{*}(i=1,2, \cdots, n)$, since $\left(\left(u_{1}{ }^{*}, Z I_{1}{ }^{*}\right),\left(u_{2}{ }^{*}, Z I_{2}{ }^{*}\right), \cdots,\left(u_{n}{ }^{*}, Z I_{n}{ }^{*}\right)\right)$ is any permutation of the $C Z I F N$ vector $\left(\left(u_{1}, Z I_{1}\right),\left(u_{2}, Z I_{2}\right), \cdots,\left(u_{n}, Z I_{n}\right)\right)$. So that

$$
\begin{aligned}
& C Z I I O W A_{\omega}\left(\left(u_{1}, Z I_{1}\right),\left(u_{2}, Z I_{2}\right), \cdots,\left(u_{n}, Z I_{n}\right)\right) \\
& =C Z I I O W A_{\omega}\left(\left(u_{1}{ }^{*}, Z I_{1}{ }^{*}\right),\left(u_{2}{ }^{*}, Z I_{2}{ }^{*}\right), \cdots,\left(u_{n}{ }^{*}, Z I_{n}{ }^{*}\right)\right) .
\end{aligned}
$$

Theorem 4.3. (Monotonicity) Assume that $\left(\left(u_{1}, Z I_{1}\right),\left(u_{2}, Z I_{2}\right), \cdots,\left(u_{n}, Z I_{n}\right)\right)$ and $\left(\left(u_{1}{ }^{*}, Z I_{1}{ }^{*}\right),\left(u_{2}{ }^{*}, Z I_{2}{ }^{*}\right), \cdots,\left(u_{n}{ }^{*}, Z I_{n}{ }^{*}\right)\right)$ are two CZIFN vectors. If $Z I_{i}<Z I_{i}{ }^{*}(i=1,2, \cdots, n)$, then

$$
\begin{aligned}
C Z I I O W & A_{\omega}\left(\left(u_{1}, Z I_{1}\right),\left(u_{2}, Z I_{2}\right), \cdots,\left(u_{n}, Z I_{n}\right)\right) \\
& <C Z I I O W A_{\omega}\left(\left(u_{1}{ }^{*}, Z I_{1}{ }^{*}\right),\left(u_{2}{ }^{*}, Z I_{2}{ }^{*}\right), \cdots,\left(u_{n}{ }^{*}, Z I_{n}{ }^{*}\right)\right) .
\end{aligned}
$$

Proof. According to Eq.(12),

$C Z I I O W A_{\omega}\left(\left(u_{1}, Z I_{1}\right),\left(u_{2}, Z I_{2}\right), \cdots,\left(u_{n}, Z I_{n}\right)\right)=\omega_{1} Z I_{\sigma(1)} \oplus \omega_{2} Z I_{\sigma(2)} \oplus \cdots \oplus \omega_{k} Z I_{\sigma(n)}$, $C Z I I O W A_{\omega}\left(\left(u_{1}{ }^{*}, Z I_{1}{ }^{*}\right),\left(u_{2}{ }^{*}, Z I_{2}{ }^{*}\right), \cdots,\left(u_{n}{ }^{*}, Z I_{n}{ }^{*}\right)\right)=\omega_{1} Z I_{\sigma(1)}^{*} \oplus \omega_{2} Z I_{\sigma(2)}^{*} \oplus \cdots \oplus \omega_{k} Z I_{\sigma(n)}^{*}$.

Since $Z I_{i}<Z I_{i}{ }^{*}(i=1,2, \cdots, n)$, we have $Z I_{\sigma(i)}<Z I_{\sigma(i)}^{*}$, then

$$
\begin{aligned}
& C Z I I O W A_{\omega}\left(\left(u_{1}, Z I_{1}\right),\left(u_{2}, Z I_{2}\right), \cdots,\left(u_{n}, Z I_{n}\right)\right) \\
& <C Z I I O W A_{\omega}\left(\left(u_{1}^{*}, Z I_{1}^{*}\right),\left(u_{2}{ }^{*}, Z I_{2}{ }^{*}\right), \cdots,\left(u_{n}{ }^{*}, Z I_{n}{ }^{*}\right)\right) .
\end{aligned}
$$

Theorem 4.4. (Idempotency) Let $\left(u_{i}, Z I_{i}\right)(i=1,2,3, \cdots, n)$ be a series of CZIIOWA pairs. If $Z I_{i}=Z I$ for $i=1,2, \cdots, n$, then

$$
C Z I I O W A_{\omega}\left(\left(u_{1}, Z I_{1}\right),\left(u_{2}, Z I_{2}\right), \cdots,\left(u_{n}, Z I_{n}\right)\right)=Z I \text {. }
$$


Proof.

$$
\begin{aligned}
C Z I I O W & A_{\omega}\left(\left(u_{1}, Z I_{1}\right),\left(u_{2}, Z I_{2}\right), \cdots,\left(u_{n}, Z I_{n}\right)\right) \\
& =\omega_{1} Z I_{\sigma(1)} \oplus \omega_{2} Z I_{\sigma(2)} \oplus \cdots \oplus \omega_{n} Z I_{\sigma(n)} \\
& =\left(\omega_{1}+\omega_{2}+\cdots+\omega_{n}\right) Z I \\
& =Z I .
\end{aligned}
$$

Theorem 4.5. (Boundedness) Let $\left(u_{i}, Z I_{i}\right)(i=1,2,3, \cdots, n)$ be a series of CZIIOWA pairs. If $Z I^{+}=\max _{1 \leq i \leq n}\left\{Z I_{i}\right\}$ and $Z I^{-}=\min _{1 \leq i \leq n}\left\{Z I_{i}\right\}$, then

$$
Z I^{-} \leq C Z I I O W A_{\omega}\left(\left(u_{1}, Z I_{1}\right),\left(u_{2}, Z I_{2}\right), \cdots,\left(u_{n}, Z I_{n}\right)\right) \leq Z I^{+} .
$$

Proof. Since $Z I^{-} \leq Z I_{i} \leq Z I^{+}$for all $i(i=1,2, \cdots, n)$, using Theorem 4.3, 4.4, we get

$$
\begin{aligned}
C Z I I O W & A_{\omega}\left(\left(u_{1}, Z I_{1}\right),\left(u_{2}, Z I_{2}\right), \cdots,\left(u_{n}, Z I_{n}\right)\right) \\
& \leq \omega_{1} Z I^{+} \oplus \omega_{2} Z I^{+} \oplus \cdots \oplus \omega_{n} Z I^{+} \\
& =Z I^{+}, \\
C Z I I O W & A_{\omega}\left(\left(u_{1}, Z I_{1}\right),\left(u_{2}, Z I_{2}\right), \cdots,\left(u_{n}, Z I_{n}\right)\right) \\
& \geq \omega_{1} Z I^{-} \oplus \omega_{2} Z I^{-} \oplus \cdots \oplus \omega_{n} Z I^{-} \\
& =Z I^{-} .
\end{aligned}
$$

So

$$
Z I^{-} \leq C Z I I O W A_{\omega}\left(\left(u_{1}, Z I_{1}\right),\left(u_{2}, Z I_{2}\right), \cdots,\left(u_{n}, Z I_{n}\right)\right) \leq Z I^{+} .
$$

Next, we shall develop CZIIOWG operator and give its relevant theorems. The proofs of the theorems are omitted, because they are similar to these of CZIIOWA operator.

Definition 9. Let $Z I_{i}=\left(A I_{i}, B I_{i}\right)=\left(\left(\mu_{A I_{i}}, \nu_{A I_{i}}\right),\left(\mu_{B I_{i}}, \nu_{B I_{i}}\right)\right) \quad(i=1,2, \cdots, n)$ be a series of CZIFNs. A CZIIOWG operator of dimention $n$ is a mapping CZIIOWG: $(R \times Z I)^{n} \rightarrow$ $Z I$, to which a weight vector $\omega=\left(\omega_{1}, \omega_{2}, \cdots, \omega_{n}\right)^{T}$ is associated to the set of second arguments of a list of $n$ pairs $\left(u_{i}, Z I_{i}\right)(i=1,2,3, \cdots, n)$ with the conditions $\omega_{i} \in[0,1]$ and $\sum_{i=1}^{n} \omega_{i}=1$. CZIIOWG is subject to the following expression:

$$
\begin{array}{r}
C Z I I O W G_{\omega}\left(\left(u_{1}, Z I_{1}\right),\left(u_{2}, Z I_{2}\right), \cdots,\left(u_{n}, Z I_{n}\right)\right) \\
=Z I_{\sigma(1)}{ }^{\omega_{1}} \otimes Z I_{\sigma(2)}{ }^{\omega_{2}} \otimes \cdots \otimes Z I_{\sigma(n)}{ }^{\omega_{n}}
\end{array}
$$


where $\sigma:(1,2, \cdots, n) \rightarrow(1,2, \cdots, n)$ is a permutation such that $u_{\sigma(1)} \geq u_{\sigma(2)} \geq \cdots \geq$ $u_{\sigma(n-1)} \geq u_{\sigma(n)}$, that is, $\left(u_{\sigma(i)}, Z I_{\sigma(i)}\right)$ is the pair in which $u_{\sigma(i)}$ is the $i$ th largest value in the set $\left\{u_{1}, u_{2}, \cdots, u_{n}\right\} . Z I_{1}, Z I_{2}, \cdots, Z I_{n}$ are induced by the ordering of the values $u_{1}, u_{2}, \cdots, u_{n}$. $u_{1}, u_{2}, \cdots, u_{n}$ are defined as the values of order-inducing variable and $Z I_{1}, Z I_{2}, \cdots, Z I_{n}$ as the values of the argument variable.

Theorem 4.6. Let $\left(u_{i}, Z I_{i}\right)(i=1,2,3, \cdots, n)$ be a series of CZIIOWG pairs. $Z I_{i}$ in $\left(u_{i}, Z I_{i}\right)$ is a CZIFN, denoted by $Z I_{i}=\left(A I_{i}, B I_{i}\right)=\left(\left(\mu_{A I_{i}}, \nu_{A I_{i}}\right),\left(\mu_{B I_{i}}, \nu_{B I_{i}}\right)\right)$. Then, their aggregated value by using the CZIIOWG operator is also a CZIFN, and

$$
\begin{aligned}
C Z I I O W & G_{\omega}\left(\left(u_{1}, Z I_{1}\right),\left(u_{2}, Z I_{2}\right), \cdots,\left(u_{n}, Z I_{n}\right)\right) \\
& =Z I_{\sigma(1)}^{\omega_{1}} \otimes Z I_{\sigma(2)}{ }^{\omega_{2}} \otimes \cdots \otimes Z I_{\sigma(n)}{ }^{\omega_{n}} \\
& =\left(\left(\prod_{i=1}^{n} \mu_{A I_{\sigma(i)}}^{\omega_{i}}, 1-\prod_{i=1}^{n}\left(1-v_{A I_{\sigma(i)}}\right)^{\omega_{i}}\right),\left(\prod_{i=1}^{n} \mu_{B I_{\sigma(i)}}^{\omega_{i}}, 1-\prod_{i=1}^{n}\left(1-v_{B I_{\sigma(i)}}\right)^{\omega_{i}}\right)\right),
\end{aligned}
$$

where $\sigma:(1,2, \cdots, n) \rightarrow(1,2, \cdots, n)$ is a permutation, and $\left(u_{\sigma(i)}, Z I_{\sigma(i)}\right)$ is the pair in which $u_{\sigma(i)}$ is the $i$ th largest value in the set $\left\{u_{1}, u_{2}, \cdots, u_{n}\right\} . Z I_{1}, Z I_{2}, \cdots, Z I_{n}$ are induced by the ordering of the values $u_{1}, u_{2}, \cdots, u_{n} . \omega=\left(\omega_{1}, \omega_{2}, \cdots, \omega_{n}\right)^{T}$ is a weight vector associated to the set of second arguments of a list of $n$ pairs $\left(u_{i}, Z I_{i}\right)(i=1,2,3, \cdots, n)$ with the conditions $\omega_{i} \in[0,1]$ and $\sum_{i=1}^{n} \omega_{i}=1$.

Theorem 4.7. (Commutativity) Let $\left(\left(u_{1}{ }^{*}, Z I_{1}{ }^{*}\right),\left(u_{2}{ }^{*}, Z I_{2}{ }^{*}\right), \cdots,\left(u_{n}{ }^{*}, Z I_{n}{ }^{*}\right)\right)$ be any permutation of the CZIFN vector $\left(\left(u_{1}, Z I_{1}\right),\left(u_{2}, Z I_{2}\right), \cdots,\left(u_{n}, Z I_{n}\right)\right)$, then

$$
\begin{aligned}
C Z I I O W G_{\omega} & \left(\left(u_{1}, Z I_{1}\right),\left(u_{2}, Z I_{2}\right), \cdots,\left(u_{n}, Z I_{n}\right)\right) \\
& =C Z I I O W G_{\omega}\left(\left(u_{1}{ }^{*}, Z I_{1}{ }^{*}\right),\left(u_{2}{ }^{*}, Z I_{2}{ }^{*}\right), \cdots,\left(u_{n}{ }^{*}, Z I_{n}{ }^{*}\right)\right) .
\end{aligned}
$$

Theorem 4.8. (Monotonicity) Assume that $\left(\left(u_{1}, Z I_{1}\right),\left(u_{2}, Z I_{2}\right), \cdots,\left(u_{n}, Z I_{n}\right)\right)$ and $\left(\left(u_{1}{ }^{*}, Z I_{1}{ }^{*}\right),\left(u_{2}{ }^{*}, Z I_{2}{ }^{*}\right), \cdots,\left(u_{n}{ }^{*}, Z I_{n}{ }^{*}\right)\right)$ are two CZIFN vectors. If $Z I_{i}<Z I_{i}{ }^{*}(i=1,2, \cdots, n)$, then

$$
\begin{aligned}
C Z I I O W & G_{\omega}\left(\left(u_{1}, Z I_{1}\right),\left(u_{2}, Z I_{2}\right), \cdots,\left(u_{n}, Z I_{n}\right)\right) \\
& <C Z I I O W G_{\omega}\left(\left(u_{1}{ }^{*}, Z I_{1}{ }^{*}\right),\left(u_{2}{ }^{*}, Z I_{2}{ }^{*}\right), \cdots,\left(u_{n}{ }^{*}, Z I_{n}{ }^{*}\right)\right) .
\end{aligned}
$$

Theorem 4.9. (Idempotency) Let $\left(u_{i}, Z I_{i}\right)(i=1,2,3, \cdots, n)$ be a series of CZIIOWG pairs. If $Z I_{i}=Z I$ for all $i=1,2, \cdots, n$, then

$$
C Z I I O W G_{\omega}\left(\left(u_{1}, Z I_{1}\right),\left(u_{2}, Z I_{2}\right), \cdots,\left(u_{n}, Z I_{n}\right)\right)=Z I
$$

Theorem 4.10. (Boundedness) Let $\left(u_{i}, Z I_{i}\right)(i=1,2,3, \cdots, n)$ be a series of CZIIOWG pairs. If $Z I^{+}=\max _{1 \leq i \leq n}\left\{Z I_{i}\right\}$ and $Z I^{-}=\min _{1 \leq i \leq n}\left\{Z I_{i}\right\}$, then

$$
Z I^{-} \leq C Z I I O W G_{\omega}\left(\left(u_{1}, Z I_{1}\right),\left(u_{2}, Z I_{2}\right), \cdots,\left(u_{n}, Z I_{n}\right)\right) \leq Z I^{+} .
$$




\section{Complete Z-intuitionistic fuzzy MUITIMOORA method with AHP}

In this section, we mainly introduce complete Z-intuitionistic fuzzy MUITIMOORA (CZIF-MA) method with AHP. First, we briefly define the distance measure of CZIFN. Then, the CZIF-MA method with AHP is proposed. Finally, we propose an approach for MCGDM based on CZIF-MA with AHP, CZIIOWA operator and CZIIOWG operator.

\subsection{Complete Z-intuitionistic fuzzy distance measure}

Distance measure is essential for ranking alternatives when using TOPSIS to deal with MCGDM problems with the CZIFN information. Traditional distance measure has not used the reliability information due to basing on former information representation. So that, inspired by [7], we propose the complete Z-intuitionistic fuzzy distance measure based on CZIFN. The distance measure of CZIFN can consider not only the intuitionistic fuzzy information but also the reliability information.

Definition 10. Let $Z I_{1}=\left(A I_{1}, B I_{1}\right)=\left(\left(\mu_{A I_{1}}, \nu_{A I_{1}}\right),\left(\mu_{B I_{1}}, \nu_{B I_{1}}\right)\right)$ and $Z I_{2}=\left(A I_{2}, B I_{2}\right)=$ $\left(\left(\mu_{A I_{2}}, \nu_{A I_{2}}\right),\left(\mu_{B I_{2}}, \nu_{B I_{2}}\right)\right)$ be any two CZIFNs. The Minkowski distance $d\left(Z I_{1}, Z I_{2}\right)$ between $Z I_{1}$ and $Z I_{2}$ is expressed as following:

$$
d\left(Z I_{1}, Z I_{2}\right)=\frac{1}{2}\left(\left|p_{Z I_{1}}{ }^{k} \mu_{A I_{1}}-p_{Z I_{2}}{ }^{k} \mu_{A I_{2}}\right|^{r}+\left|p_{Z I_{1}}{ }^{k}\left(1-v_{A I_{1}}\right)-p_{Z I_{2}}{ }^{k}\left(1-v_{A I_{2}}\right)\right|^{r}\right)^{\frac{1}{r}},
$$

where $r \geq 1$ and $k \geq 0$ are parameters, and $p_{Z I_{i}}=\frac{\mu_{B I_{i}}}{\mu_{B I_{i}}+v_{B I_{i}}} \cdot p_{Z I_{i}}$ is a standard reliability measure of $Z I_{i}(i=1,2)$.

The change of the size of $k$, a tunable parameter in Eq.(24), can control the effect of reliability in distance measure. Next, we will discuss how the size of $k$ affects the distance measure of CZIFNs. The proofs of Theorem 5.1-5.4 are similar to the proofs of Theorem 3.3-3.6, so we omit them.

Theorem 5.1. When $k=0$, it means that reliability information in distance measure has no effect.

Theorem 5.2. When $0<k<1$, it means that the effect of reliability information in distance measure is strong.

Theorem 5.3. When $k=1$, it means that the effect of reliability information in distance measure is neutral.

Theorem 5.4. When $k>1$, it means that the effect of reliability information in distance measure is weak.

Minkowski distance, as a definition of a group of distances, can be expressed by different parameter values. When $r=1$ and $r=2$, Minkowski distance can degenerate into Hamming and Euclidean distance respectively.

Remark 5.1. When $r=1$, then Eq.(24) is a Hamming distance:

$$
d\left(Z I_{1}, Z I_{2}\right)=\frac{1}{2}\left(\left|p_{Z I_{1}}{ }^{k} \mu_{A I_{1}}-p_{Z I_{2}}{ }^{k} \mu_{A I_{2}}\right|+\left|p_{Z I_{1}}{ }^{k}\left(1-v_{A I_{1}}\right)-p_{Z I_{2}}{ }^{k}\left(1-v_{A I_{2}}\right)\right|\right) \text {. }
$$


Remark 5.2. When $r=2$, then Eq.(24) is an Euclidean distance:

$$
d\left(Z I_{1}, Z I_{2}\right)=\frac{1}{2}\left(\left|p_{Z I_{1}}{ }^{k} \mu_{A I_{1}}-p_{Z I_{2}}{ }^{k} \mu_{A I_{2}}\right|^{2}+\left|p_{Z I_{1}}{ }^{k}\left(1-v_{A I_{1}}\right)-p_{Z I_{2}}{ }^{k}\left(1-v_{A I_{2}}\right)\right|^{2}\right)^{\frac{1}{2}} .
$$

Remark 5.3. When $\nu_{B I}=1-\mu_{B I}$, then Eq.(24) will be simplified to the distance measure of AZIFNs.

Remark 5.4. When $\nu_{A I}=1-\mu_{A I}$, then Eq.(24) will be simplified to the distance measure of BZIFNs.

Remark 5.5. When $\nu_{A I}=1-\mu_{A I}$ and $\nu_{B I}=1-\mu_{B I}$, then Eq.(24) will be simplified to the distance measure of Z-numbers.

Remark 5.6. When $\mu_{B I}=1$, then Eq.(24) will be simplified to the distance measure of IFNs.

Theorem 5.5. Let $Z I_{1}=\left(A I_{1}, B I_{1}\right)=\left(\left(\mu_{A I_{1}}, \nu_{A I_{1}}\right),\left(\mu_{B I_{1}}, \nu_{B I_{1}}\right)\right), Z I_{2}=\left(A I_{2}, B I_{2}\right)=$ $\left(\left(\mu_{A I_{2}}, \nu_{A I_{2}}\right),\left(\mu_{B I_{2}}, \nu_{B I_{2}}\right)\right)$ and $Z I_{3}=\left(A I_{3}, B I_{3}\right)=\left(\left(\mu_{A I_{3}}, \nu_{A I_{3}}\right),\left(\mu_{B I_{3}}, \nu_{B I_{3}}\right)\right)$ be any three CZIFNs. Then:

(1) $0 \leq d\left(Z I_{1}, Z I_{2}\right) \leq 1$

(2) $d\left(Z I_{1}, Z I_{1}\right)=0$

(3) $d\left(Z I_{1}, Z I_{2}\right)=d\left(Z I_{2}, Z I_{1}\right)$;

(4) $d\left(Z I_{1}, Z I_{2}\right)+d\left(Z I_{2}, Z I_{3}\right) \geq d\left(Z I_{1}, Z I_{3}\right)$.

\section{Proof.}

(1)

$$
0 \leq d\left(Z I_{1}, Z I_{2}\right)=\frac{1}{2}\left(\left|p_{Z I_{1}}{ }^{k} \mu_{A I_{1}}-p_{Z I_{2}}{ }^{k} \mu_{A I_{2}}\right|^{r}+\left|p_{Z I_{1}}{ }^{k}\left(1-v_{A I_{1}}\right)-p_{Z I_{2}}{ }^{k}\left(1-v_{A I_{2}}\right)\right|^{r}\right)^{\frac{1}{r}} \leq 1 ;
$$

$$
d\left(Z I_{1}, Z I_{1}\right)=\frac{1}{2}\left(\left|p_{Z I_{1}}{ }^{k} \mu_{A I_{1}}-p_{Z I_{1}}{ }^{k} \mu_{A I_{1}}\right|^{r}+\left|p_{Z I_{1}}{ }^{k}\left(1-v_{A I_{1}}\right)-p_{Z I_{1}}{ }^{k}\left(1-v_{A I_{1}}\right)\right|^{r}\right)^{\frac{1}{r}}=0 ;
$$

$$
\begin{aligned}
d\left(Z I_{1}, Z I_{2}\right) & =\frac{1}{2}\left(\left|p_{Z I_{1}}{ }^{k} \mu_{A I_{1}}-p_{Z I_{2}}{ }^{k} \mu_{A I_{2}}\right|^{r}+\left|p_{Z I_{1}}{ }^{k}\left(1-v_{A I_{1}}\right)-p_{Z I_{2}}{ }^{k}\left(1-v_{A I_{2}}\right)\right|^{r}\right)^{\frac{1}{r}} \\
& =\frac{1}{2}\left(\left|p_{Z I_{2}}{ }^{k} \mu_{A I_{2}}-p_{Z I_{1}}{ }^{k} \mu_{A I_{1}}\right|^{r}+\left|p_{Z I_{2}}{ }^{k}\left(1-v_{A I_{2}}\right)-p_{Z I_{1}}{ }^{k}\left(1-v_{A I_{1}}\right)\right|^{r}\right)^{\frac{1}{r}} \\
& =d\left(Z I_{2}, Z I_{1}\right) ;
\end{aligned}
$$

(4) According to the Minkowski inequality $\left(\sum_{i=1}^{n}\left|x_{i}+y_{i}\right|^{r}\right)^{\frac{1}{r}} \leq\left(\sum_{i=1}^{n}\left|x_{i}\right|^{r}\right)^{\frac{1}{r}}+\left(\sum_{i=1}^{n}\left|y_{i}\right|^{r}\right)^{\frac{1}{r}}$, where $x_{i}, y_{i} \in R, \quad i=1,2, \cdots, n$ and $r \geq 1$, we have 


$$
\begin{aligned}
d & \left(Z I_{1}, Z I_{2}\right)+d\left(Z I_{2}, Z I_{3}\right) \\
= & \frac{1}{2}\left(\begin{array}{c}
\left(\left|p_{Z I_{1}}{ }^{k} \mu_{A I_{1}}-p_{Z I_{2}}{ }^{k} \mu_{A I_{2}}\right|^{r}+\left|p_{Z I_{1}}{ }^{k}\left(1-v_{A I_{1}}\right)-p_{Z I_{2}}{ }^{k}\left(1-v_{A I_{2}}\right)\right|^{r}\right)^{\frac{1}{r}} \\
+\left(\left|p_{Z I_{2}}{ }^{k} \mu_{A I_{2}}-p_{Z I_{3}}{ }^{k} \mu_{A I_{3}}\right|^{r}+\left|p_{Z I_{2}}{ }^{k}\left(1-v_{A I_{2}}\right)-p_{Z I_{3}}{ }^{k}\left(1-v_{A I_{3}}\right)\right|^{r}\right)^{\frac{1}{r}}
\end{array}\right) \\
\geq & \frac{1}{2}\left(\begin{array}{c}
\mid p_{Z I_{1}}{ }^{k} \mu_{A I_{1}}-p_{Z I_{2}}{ }^{k} \mu_{A I_{2}}+p_{Z I_{2}}{ }^{k} \mu_{A I_{2}}-p_{Z I_{3}}{ }^{k} \mu_{A I_{3}}{ }^{r}+ \\
\left|p_{Z I_{1}}{ }^{k}\left(1-v_{A I_{1}}\right)-p_{Z I_{2}}{ }^{k}\left(1-v_{A I_{2}}\right)+p_{Z I_{2}}{ }^{k}\left(1-v_{A I_{2}}\right)-p_{Z I_{3}}{ }^{k}\left(1-v_{A I_{3}}\right)\right|^{r}
\end{array}\right) \\
= & \frac{1}{2}\left(\left|p_{Z I_{1}}{ }^{k} \mu_{A I_{1}}-p_{Z I_{3}}{ }^{k} \mu_{A I_{3}}\right|^{r}+\left|p_{Z I_{1}}{ }^{k}\left(1-v_{A I_{1}}\right)-p_{Z I_{3}}{ }^{k}\left(1-v_{A I_{3}}\right)\right|^{r}\right)^{\frac{1}{r}} \\
= & d\left(Z I_{1}, Z I_{3}\right) .
\end{aligned}
$$

Remark 5.7. Let $Z I_{1}=\left(A I_{1}, B I_{1}\right)=\left(\left(\mu_{A I_{1}}, \nu_{A I_{1}}\right),\left(\mu_{B I_{1}}, \nu_{B I_{1}}\right)\right), Z I_{2}=\left(A I_{2}, B I_{2}\right)=$ $\left(\left(\mu_{A I_{2}}, \nu_{A I_{2}}\right),\left(\mu_{B I_{2}}, \nu_{B I_{2}}\right)\right)$ and $Z I_{3}=\left(A I_{3}, B I_{3}\right)=\left(\left(\mu_{A I_{3}}, \nu_{A I_{3}}\right),\left(\mu_{B I_{3}}, \nu_{B I_{3}}\right)\right)$ be any three CZIFNs. Then:

(1) When $0<r<1$, then

$$
d\left(Z I_{1}, Z I_{2}\right)+d\left(Z I_{2}, Z I_{3}\right) \geq \frac{1}{2 M} d\left(Z I_{1}, Z I_{3}\right)
$$

where $M=\max \left\{\left|p_{Z I_{1}}{ }^{k} \mu_{A I_{1}}-p_{Z I_{2}}{ }^{k} \mu_{A I_{2}}\right|,\left|p_{Z I_{1}}{ }^{k}\left(1-v_{A I_{1}}\right)-p_{Z I_{2}}{ }^{k}\left(1-v_{A I_{2}}\right)\right|, \mid p_{Z I_{2}}{ }^{k} \mu_{A I_{2}}-\right.$ $\left.p_{Z I_{3}}{ }^{k} \mu_{A I_{3}}|,| p_{Z I_{2}}{ }^{k}\left(1-v_{A I_{2}}\right)-p_{Z I_{3}}{ }^{k}\left(1-v_{A I_{3}}\right) \mid\right\}$.

(2) When $r<-1$, then

$$
d\left(Z I_{1}, Z I_{2}\right)+d\left(Z I_{2}, Z I_{3}\right) \geq \frac{2^{2 r}\left(d\left(Z I_{1}, Z I_{3}\right)\right)^{2 r+1}}{\left|p_{Z I_{1}}{ }^{k} \mu_{A I_{1}}-p_{Z I_{3}}{ }^{k} \mu_{A I_{3}}\right|^{r} \cdot \mid p_{Z I_{1}}{ }^{k}\left(1-v_{A I_{1}}\right)-p_{Z I_{3}}{ }^{k}\left(1-v_{A I_{3}}\right)^{r}} .
$$

Proof.

(1) According to the inequality[40] $\frac{1}{2 M}\left(\sum_{i=1}^{n}\left|x_{i}+y_{i}\right|^{r}\right)^{\frac{1}{r}} \leq\left(\sum_{i=1}^{n}\left|x_{i}\right|^{r}\right)^{\frac{1}{r}}+\left(\sum_{i=1}^{n}\left|y_{i}\right|^{r}\right)^{\frac{1}{r}}$, where $x_{i}, y_{i} \in R, M=\max _{i}\left\{x_{i}, y_{i}\right\}, i=1,2, \cdots, n$ and $0<r<1$, we have

$$
\begin{aligned}
& d\left(Z I_{1}, Z I_{2}\right)+d\left(Z I_{2}, Z I_{3}\right) \\
&= \frac{1}{2}\left(\begin{array}{c}
\left(\left|p_{Z I_{1}}{ }^{k} \mu_{A I_{1}}-p_{Z I_{2}}{ }^{k} \mu_{A I_{2}}\right|^{r}+\left|p_{Z I_{1}}{ }^{k}\left(1-v_{A I_{1}}\right)-p_{Z I_{2}}{ }^{k}\left(1-v_{A I_{2}}\right)\right|^{r}\right)^{\frac{1}{r}} \\
+\left(\left|p_{Z I_{2}}{ }^{k} \mu_{A I_{2}}-p_{Z I_{3}}{ }^{k} \mu_{A I_{3}}\right|^{r}+\left|p_{Z I_{2}}{ }^{k}\left(1-v_{A I_{2}}\right)-p_{Z I_{3}}{ }^{k}\left(1-v_{A I_{3}}\right)\right|^{r}\right)^{\frac{1}{r}}
\end{array}\right) \\
& \geq \frac{1}{4 M}\left(\begin{array}{c}
\left|p_{Z I_{1}}{ }^{k} \mu_{A I_{1}}-p_{Z I_{2}}{ }^{k} \mu_{A I_{2}}+p_{Z I_{2}}{ }^{k} \mu_{A I_{2}}-p_{Z I_{3}}{ }^{k} \mu_{A I_{3}}\right|^{r}+ \\
\left|p_{Z I_{1}}{ }^{k}\left(1-v_{A I_{1}}\right)-p_{Z I_{2}}{ }^{k}\left(1-v_{A I_{2}}\right)+p_{Z I_{2}}{ }^{k}\left(1-v_{A I_{2}}\right)-p_{Z I_{3}}{ }^{k}\left(1-v_{A I_{3}}\right)\right|^{r}
\end{array}\right){ }^{\frac{1}{r}} \\
&=\frac{1}{4 M}\left(\left|p_{Z I_{1}}{ }^{k} \mu_{A I_{1}}-p_{Z I_{3}}{ }^{k} \mu_{A I_{3}}\right|^{r}+\left|p_{Z I_{1}}{ }^{k}\left(1-v_{A I_{1}}\right)-p_{Z I_{3}}{ }^{k}\left(1-v_{A I_{3}}\right)\right|^{r}\right)^{\frac{1}{r}} \\
&=\frac{1}{2 M} d\left(Z I_{1}, Z I_{3}\right),
\end{aligned}
$$


where $M=\max \left\{\left|p_{Z I_{1}}{ }^{k} \mu_{A I_{1}}-p_{Z I_{2}}{ }^{k} \mu_{A I_{2}}\right|,\left|p_{Z I_{1}}{ }^{k}\left(1-v_{A I_{1}}\right)-p_{Z I_{2}}{ }^{k}\left(1-v_{A I_{2}}\right)\right|, \mid p_{Z I_{2}}{ }^{k} \mu_{A I_{2}}-\right.$ $\left.p_{Z I_{3}}{ }^{k} \mu_{A I_{3}}|,| p_{Z I_{2}}{ }^{k}\left(1-v_{A I_{2}}\right)-p_{Z I_{3}}{ }^{k}\left(1-v_{A I_{3}}\right) \mid\right\}$.

(2) According to the inequality[40] $\sum_{i=1}^{n}\left|x_{i}+y_{i}\right|^{r} \sum_{i=1}^{n} \frac{1}{\left|x_{i}+y_{i}\right|^{r}}\left(\sum_{i=1}^{n}\left|x_{i}+y_{i}\right|^{r}\right)^{\frac{1}{r}} \leq\left(\sum_{i=1}^{n}\left|x_{i}\right|^{r}\right)^{\frac{1}{r}}+$ $\left(\sum_{i=1}^{n}\left|y_{i}\right|^{r}\right)^{\frac{1}{r}}$, where $x_{i}, y_{i} \in R, \quad i=1,2, \cdots, n$ and $r<-1$, we have

$$
\begin{aligned}
& d\left(Z I_{1}, Z I_{2}\right)+d\left(Z I_{2}, Z I_{3}\right) \\
& =\frac{1}{2}\left(\begin{array}{c}
\left(\left|p_{Z I_{1}}{ }^{k} \mu_{A I_{1}}-p_{Z I_{2}}{ }^{k} \mu_{A I_{2}}\right|^{r}+\left|p_{Z I_{1}}{ }^{k}\left(1-v_{A I_{1}}\right)-p_{Z I_{2}}{ }^{k}\left(1-v_{A I_{2}}\right)\right|^{r}\right)^{\frac{1}{r}} \\
+\left(\left|p_{Z I_{2}}{ }^{k} \mu_{A I_{2}}-p_{Z I_{3}}{ }^{k} \mu_{A I_{3}}\right|^{r}+\left|p_{Z I_{2}}{ }^{k}\left(1-v_{A I_{2}}\right)-p_{Z I_{3}}{ }^{k}\left(1-v_{A I_{3}}\right)\right|^{r}\right)^{\frac{1}{r}}
\end{array}\right) \\
& \geq \frac{1}{2}\left(\begin{array}{c}
\left(\left|p_{Z I_{1}}{ }^{k} \mu_{A I_{1}}-p_{Z I_{3}}{ }^{k} \mu_{A I_{3}}\right|^{r}+\left|p_{Z I_{1}}{ }^{k}\left(1-v_{A I_{1}}\right)-p_{Z I_{3}}{ }^{k}\left(1-v_{A I_{3}}\right)\right|^{r}\right)^{\frac{1}{r}+1} \\
\cdot\left(\frac{1}{\left|p_{Z I_{1}}{ }^{k} \mu_{A I_{1}}-p_{Z I_{3}}{ }^{k} \mu_{A I_{3}}\right|^{r}}+\frac{1}{\left|p_{Z I_{1}}{ }^{k}\left(1-v_{A I_{1}}\right)-p_{Z I_{3}}{ }^{k}\left(1-v_{A I_{3}}\right)\right|^{r}}\right)
\end{array}\right) \\
& =\frac{2^{2 r}\left(d\left(Z I_{1}, Z I_{3}\right)\right)^{2 r+1}}{\left|p_{Z I_{1}}{ }^{k} \mu_{A I_{1}}-p_{Z I_{3}}{ }^{k} \mu_{A I_{3}}\right|^{r} \cdot\left|p_{Z I_{1}}{ }^{k}\left(1-v_{A I_{1}}\right)-p_{Z I_{3}}{ }^{k}\left(1-v_{A I_{3}}\right)\right|^{r}} .
\end{aligned}
$$

\subsection{Improved MULTIMOORA method with AHP based on CZIFN}

For a MCGDM problem with CZIFN, AZIFN, BZIFN and IFN information, let $A=$ $\left\{a_{i} \mid i=1,2, \cdots, m\right\}$ and $C=\left\{c_{j} \mid j=1,2, \cdots, n\right\}$ be two finite sets of alternatives and criteria respectively. $\omega=\left(\omega_{1}, \omega_{2}, \cdots, \omega_{n}\right)^{T}$, with the conditions $\omega_{j} \in[0,1]$ and $\sum_{j=1}^{n} \omega_{j}=1$, is a weight vector associated with the criteria. Let $q$ be the number of benefit criteria and $n-q$ be the number of cost criteria. $\left\{b_{h} \mid h=1,2, \cdots, l\right\}$ is a set of DMs with the weight vector $\tilde{\omega}=\left(\tilde{\omega}_{1}, \tilde{\omega}_{2}, \cdots, \tilde{\omega}_{l}\right)^{T}$ satisfying $\tilde{\omega}_{h} \in[0,1]$ and $\sum_{h=1}^{l} \tilde{\omega}_{h}=1$. Suppose that $D^{(h)}=\left[I_{i j}^{(h)}\right]_{m \times n}$ is a mixed individual decision matrix, where $I_{i j}^{(h)}$ is a CZIFN, AZIFN, BZIFN, or IFN, which indicates the evaluation value of the alternative $a_{i}(i=1,2, \cdots, m)$ about the criteria $c_{j}(j=1,2, \cdots, n)$ given by the DM $b_{h}(h=1,2, \cdots, l)$. First of all, according to Theorem 3.1, convert all evaluation information $I_{i j}^{(h)}$ into CZIFN, denoted by $Z I_{i j}^{(h)}=\left(A I_{i j}^{(h)}, B I_{i j}^{(h)}\right)=$ $\left(\left(\mu_{A I_{i j}^{(h)}}, v_{A I_{i j}^{(h)}}\right),\left(\mu_{B I_{i j}^{(h)}}, v_{A I_{i j}^{(h)}}\right)\right)$. Then, based on CZIIOWA or CZIIOWG operator, group decision matrix $D=\left[Z I_{i j}\right]_{m \times n}$ is determined by:

$$
D=\left[\begin{array}{ccccc}
Z I_{11} & \cdots & Z I_{1 j} & \cdots & Z I_{1 n} \\
\vdots & \ddots & \vdots & \ddots & \vdots \\
Z I_{i 1} & \cdots & Z I_{i j} & \cdots & Z I_{i n} \\
\vdots & \ddots & \vdots & \ddots & \vdots \\
Z I_{m 1} & \cdots & Z I_{m j} & \cdots & Z I_{m n}
\end{array}\right]
$$


Because traditional MULTIMOORA method has many shortcomings, refered in Introduction, we improve this method from three aspects. First, we develop it based on CZIFN, which considers both the intuitionistic fuzzy and reliability information. Besides, we extend the RP model, so that, this model can consider both the profile and risk of alternatives. Moreover, we are committed to integrating AHP into this method to fuse the three rankings into a single one.

Convert all the criteria into benefit criteria by Eq. (27). After this process, we get the standard decision matrix $\tilde{D}=\left[Z \tilde{I}_{i j}\right]_{m \times n}$.

$$
\tilde{Z I}_{i j}=\left\{\begin{array}{l}
\left(\left(\mu_{A I_{i j}}, v_{A I_{i j}}\right),\left(\mu_{B I_{i j}}, v_{B I_{i j}}\right)\right), j \leq q \\
\left(\left(v_{A I_{i j}}, \mu_{A I_{i j}}\right),\left(\mu_{B I_{i j}}, v_{B I_{i j}}\right)\right), j>q .
\end{array}\right.
$$

\subsubsection{Subordinate rankings of CZIF-MA}

1). Ratio system model of CZIFN (CZIFN-RS): Ratio system is a compensatory model, which adopts the arithmetic weighted aggregation operator. It indicates that the large values of an alternative can completely compensate the same degree of small values[41]. Therefore, $\mathrm{RS}$ model pays more attention to the average performance of the alternatives on each criterion. CZIFN-RS model is given as follows:

$$
y_{1}\left(a_{i}\right)=\sum_{j=1}^{n} \omega_{j} \tilde{Z}_{i j}
$$

CZIFN-RS model is a benefit criterion, in other words, bigger the $y_{1}\left(a_{i}\right)$ is, higher the ranking of $a_{i}$ should be. Obviously, $y_{1}\left(a_{i}\right)(i=1,2, \cdots, m)$ are CZIFNs, so we rank $y_{1}\left(a_{i}\right)$ $(i=1,2, \cdots, m)$ by the method we proposed in Section 3 and the alternatives are ranked in descending order of $y_{1}\left(a_{i}\right)$.

2). Reference point model of CZIFN (CZIFN-RP): In reference point approach, the best alternative is the one that its worst value in respect of all criteria is not very bad[41]. We improve RP model as following expression:

$$
y_{2}\left(a_{i}\right)=\max _{j} \omega_{j} d\left(\tilde{Z}^{+}, \tilde{Z I_{i j}}\right)-\min _{j} \omega_{j} d\left(\tilde{Z I}^{-}, \tilde{I}_{i j}\right)
$$

where

$$
\begin{aligned}
\tilde{I}^{+} & =\left(\left(\max _{i} \tilde{\mu}_{A I_{i j}}, \min _{i} \tilde{\nu}_{A I_{i j}}\right),\left(\max _{i} \tilde{\mu}_{B I_{i j}}, \min _{i} \tilde{\nu}_{B I_{i j}}\right)\right) \\
Z I^{-} & =\left(\left(\min _{i} \tilde{\mu}_{A I_{i j}}, \max _{i} \tilde{\nu}_{A I_{i j}}\right),\left(\min _{i} \tilde{\mu}_{B I_{i j}}, \max _{i} \tilde{\nu}_{B I_{i j}}\right)\right)
\end{aligned}
$$

CZIFN-RP model consider the profile and risk of alternatives simultaneously. CZIFN-RP model is a cost criterion, so the alternatives are ranked in ascending order of $y_{2}\left(a_{i}\right)$.

3). Full-multiplicative form model of CZIFN (CZIFN-FMF) : Full Multiplicative Form, which adopts the geometric weighted aggregation operator, is an incompletely-compensatory model. It indicates that the large values of an alternative can not completely compensate 
the same degree of small values[41]. Thus, FMF system pays more attention to whether the alternative is outstanding under some criteria. This model can be represented as follows:

$$
y_{3}\left(a_{i}\right)=\prod_{j=1}^{n} \tilde{Z}_{i j}{ }^{\omega_{j}} .
$$

CZIFN-FMF model is a benefit criterion, so bigger the $y_{3}\left(a_{i}\right)$ is, higher the ranking of $a_{i}$ should be. The alternatives are ranked in descending order of $y_{3}\left(a_{i}\right)$.

\subsubsection{Ranking aggregation based on AHP}

After obtaining the subordinate rankings, we need to integrate these rankings to obtain the final ranking of alternatives. As discussed by Brauers et al.[29], we could obtain an integrative ranking that is more robust than each subordinate ranking by aggregating multiple subordinate rankings. As we know, the three subordinate rankings focus on the different aspects of the alternatives. However, in the traditional MULTIMOORA method, three subordinate utility values are only integrated by the dominance theory, which does not take into account the preferences of DMs. Therefore, we introduce the AHP method to determine the weights of three subordinate rankings, and the weighted average of the subordinate rankings is used as the final sorting result.

First, given that $s_{f}\left(a_{i}\right)(f=1,2,3)$ are the corresponding sort values of the utility values $y_{f}\left(a_{i}\right)(f=1,2,3)$. Then, we construct the sort value matrix $D(s)=\left(s_{f}\left(a_{i}\right)\right)_{m \times 3}$. The procedure of ranking aggregation based on AHP is given as follows:

Table 1: Saaty's scale

\begin{tabular}{ll}
\hline Scores & Equivalent linguistic judgment \\
\hline 1 & Equally important \\
$3-1 / 3$ & Moderately more/less important \\
$5-1 / 5$ & Fairly more/less important \\
$7-1 / 7$ & Strongly more/less important \\
$9-1 / 9$ & Extremely more/less important \\
$2-1 / 2,4-1 / 4,6-1 / 6,8-1 / 8$ & Intermediate values \\
\hline
\end{tabular}

1). Construct judgment matrix. DMs compare each subordinate ranking against the others using a nine point Saaty's scale[42] shown in Table 1. Then, the judgment matrix is obtained as following:

$$
J^{(h)}=\left[\begin{array}{lll}
e_{11}^{(h)} & e_{12}^{(h)} & e_{13}^{(h)} \\
e_{21}^{(h)} & e_{22}^{(h)} & e_{23}^{(h)} \\
e_{31}^{(h)} & e_{32}^{(h)} & e_{33}^{(h)}
\end{array}\right](h=1,2, \cdots, l)
$$

where $e_{f g}^{(h)}(f=1,2,3 ; g=1,2,3)$ represents relative importance of subordinate ranking $f$ to subordinate ranking $g$ given by the $\mathrm{DM} b_{h}$, and $e_{f g}^{(h)}=1 / e_{g f}^{(h)}, e_{f f}^{(h)}=1$. 
2). Check consistency of the judgment matrix. The consistency ratio(CR) can be obtained by Eq.(31):

$$
C R=\frac{\left(\lambda_{\max }-3\right)}{2 R I},
$$

where $\lambda_{\max }$ is the maximum eigenvalue of $J^{(h)}$, and $R I=0.58$, which represents a random index. If the value of $C R$ is lower than 0.1 , the level of consistency is acceptable, otherwise the judgment matrix need to be adjusted.

3). Calculate the weight of subordinate rankings. The weight vector $\left.\overline{\widehat{\omega}^{(h)}}=\overline{\left(\widehat{\omega}_{1}^{(h)}\right.}, \overline{\widehat{\omega}_{2}^{(h)}}, \overline{\widehat{\omega}_{3}^{(h)}}\right)^{T}$ of three subordinate rankings can be calculated through Eq.(32):

$$
J^{(h)} \overline{\widehat{\omega}^{(h)}}=\lambda_{\max } \overline{\widehat{\omega}^{(h)}}
$$

where $\lambda_{\max }$ is the maximum eigenvalue of $J^{(h)}$. Then, normalized the weight vector by Eq.(33):

$$
\widehat{\omega}_{f}^{(h)}=\frac{\overline{\widehat{\omega}_{f}^{(h)}}}{\sum_{g=1}^{3} \overline{\widehat{\omega}_{g}^{(h)}}} \quad(f=1,2,3),
$$

The final weight of the subordinate rankings is the weighted average of the weights given by each DM, calculated by Eq.(34):

$$
\widehat{\omega}_{f}=\sum_{h=1}^{l} \tilde{\omega}_{h} \widehat{\omega}_{f}^{(h)} \quad(f=1,2,3) .
$$

4). Compute the final ranking. Aggregate the three groups of sort values to get the final results based on Eq.(35):

$$
F\left(a_{i}\right)=\widehat{\omega}_{1} s_{1}\left(a_{i}\right)+\widehat{\omega}_{2} s_{2}\left(a_{i}\right)+\widehat{\omega}_{3} s_{3}\left(a_{i}\right) .
$$

The final ranking of alternatives is determined in ascending order of $F\left(a_{i}\right)(i=1,2, \cdots, m)$.

5.3. An approach for MCGDM based on CZIIOWA operator, CZIIOWG operator, and CZIF$M A$ method with $A H P$

We summarize an approach for MCGDM based on CZIIOWA operator, CZIIOWG operator, and CZIF-MA method with AHP. The process of approach is visualized in Figure 1, and the specific procedure is as follows:

Step 1. Collect evaluation information, denoted by $I_{i j}^{(h)}(i=1,2, \cdots, m ; j=1,2, \cdots, n ; h=$ $1,2, \cdots, l)$, from the experts $b_{h}$ on alternatives $a_{i}(i=1,2, \cdots, m)$ with respect to criteria $c_{j}$ in CZIFN, AZIFN, BZIFN or IFN, and obtain the mixed individual decision matrices $D^{(h)}=\left[I_{i j}^{(h)}\right]_{m \times n}$.

Step 2. Convert the expressions of all evaluation information to CZIFN according to Theorem 3.1, and obtain the CZIFN individual decision matrices $\widehat{D}^{(h)}=\left[Z I_{i j}^{(h)}\right]_{m \times n}$. 
Step 3. Compute the collective opinion matrix, denoted by $D=\left[Z I_{i j}\right]_{m \times n}$, with the individual decision matrices by CZIIOWA or CZIIOWG operator.

Step 4. Convert all the criteria to be positive by Eq.(27), and get the normalized decision matrix $\tilde{D}=\left[\tilde{I}_{i j}\right]_{m \times n}$.

Step 5. Obtain the first utility value $y_{1}\left(a_{i}\right)(i=1,2, \cdots, m)$ by Eq.(28). The alternatives are ranked in descending order of $y_{1}\left(a_{i}\right)$.

Step 6. Calculate the second utility value $y_{2}\left(a_{i}\right)(i=1,2, \cdots, m)$ by Eq.(29). The alternatives are ranked in ascending order of $y_{2}\left(a_{i}\right)$.

Step 7. Compute the third utility value $y_{3}\left(a_{i}\right)(i=1,2, \cdots, m)$ by Eq.(30). The alternatives are ranked in descending order of $y_{3}\left(a_{i}\right)$.

Step 8. Determine the weight of three subordinate rankings by AHP method.

Step 9.Aggregate the three groups of sort values to get the final results $F\left(a_{i}\right)(i=1,2, \cdots, m)$ based on Eq.(35). The final ranking of alternatives is determined in ascending order of $F\left(a_{i}\right)$ $(i=1,2, \cdots, m)$.

Step 10. End 


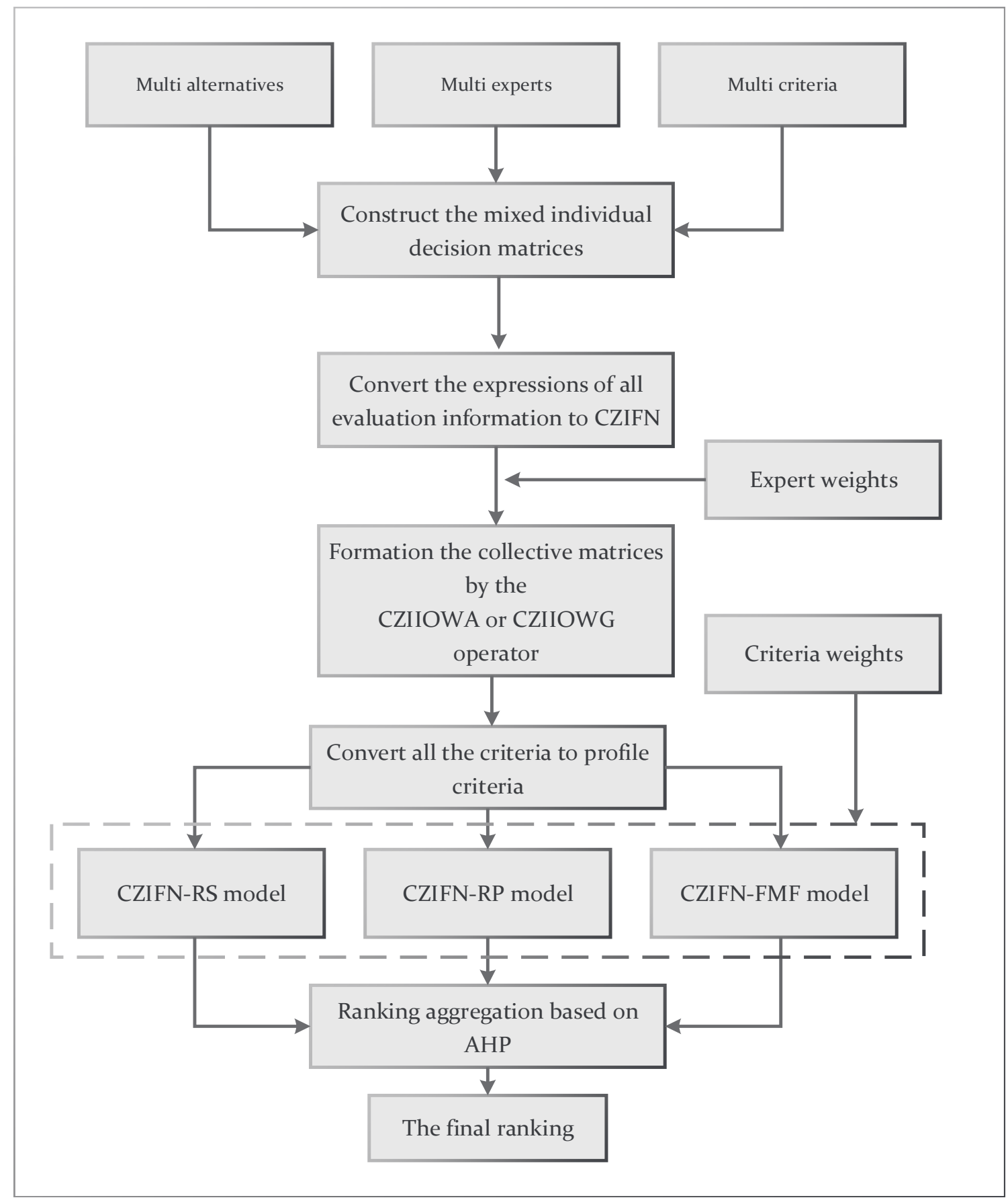

Figure 1: Process of CZIF-MA method for MCGDM problem

\section{Numerical example and comparative analysis}

In this section, we solve site selecting of Fangcang shelter hospital for COVID-19 by the proposed method. 


\subsection{Site selecting of Fangcang shelter hospital for COVID-19}

Suppose that many COVID-19 patients arise in a particular place, and there is a continuous growth trend, and it is necessary to build a Fangcang shelter hospital to deal with it. After the initial screening, 4 locations $\left\{a_{1}, a_{2}, a_{3}, a_{4}\right\}$ meet the requirements of reconstruction, and three DMs $\left\{b_{1}, b_{2}, b_{3}\right\}$ will evaluate the locations from the following four criteria:

(1) Traffic conditions $\left(c_{1}\right)$ : The Fangcang shelter hospital's location should be convenient enough and far away from the city's crowded activity areas such as business district, school and other crowded urban areas[43]. This is a benefit criterion.

(2) Peripheral supporting facilities $\left(c_{2}\right)$ : The barrier-free measures around the site should be complete. Moreover, selected sites can reserve a place for temporary parking and material turnover, and have complete security facilities and drainage systems, etc[43]. This is a benefit criterion.

(3) Venue capacity $\left(c_{3}\right)$ : The size of selected sites should meet the needs of epidemic development which is predicted by DMs based on present data of epidemic. This is a benefit criterion.

(4) Retrofit cost $\left(c_{4}\right)$ : Once the essential functions are guaranteed, the complexity and cost of transformation should be reduced as much as possible. This is a cost criterion.

The weight vector of criteria is $\omega=(0.25,0.35,0.2,0.2)$, and the weight vector of DMs is $\tilde{\omega}=(0.4,0.35,0.25)$. We use hamming distance to calculate CZIFN-RP model. We take $k=1$ in this section.

Step 1. Collect the evaluation information and obtain the mixed individual decision matrices. The decision matrices are shown in Table 2, 3, 4;

Step 2. Convert the expressions of all evaluation information to CZIFN according to Theorem 3.1, and obtain the CZIFN individual decision matrices;

Step 3. Compute the collective opinion matrix with the individual matrices by CZIIOWA operator;

Step 4. Convert all the criteria to be positive by Eq.(27), and get the normalized decision matrix $\widetilde{D}$, which is shown in Table 5;

Step 5. Obtain the first utility value $y_{1}\left(a_{i}\right)(i=1,2,3,4)$ by Eq.(28). The alternatives are ranked in descending order of $y_{1}\left(a_{i}\right)$. Table 6 presents the results;

Step 6. Calculate the second utility value $y_{2}\left(a_{i}\right)(i=1,2,3,4)$ by Eq.(29). The alternatives are ranked in ascending order of $y_{2}\left(a_{i}\right)$. The results are presented in Table 7;

Step 7. Compute the third utility value $y_{3}\left(a_{i}\right)(i=1,2,3,4)$ by Eq.(30). The alternatives are ranked in descending order of $y_{3}\left(a_{i}\right)$. Table 8 shows the results;

Step 8. Determine the weight of three subordinate rankings by AHP method. The judgment matrix after consistency test is shown in Table 9. The weight vector of three subordinate ranking is $\widehat{\omega}=(0.2304,0.6322,0.1374)$;

Step 9. Aggregate the three groups of sort values to get the final results. We can see the results in Table 10. The final ranking is $a_{1} \succ a_{4} \succ a_{2} \succ a_{3}$, and the best option is $a_{1}$;

Step 10. End. 
Table 2: Mixed Individual Decision Matrix $D^{(1)}$

$\begin{array}{lllll} & c_{1} & c_{2} & c_{3} & c_{4} \\ a_{1} & ((0.6,0.3), 0.8) & ((0.7,0.2),(0.5,0.2)) & ((0.5,0.3), 0.6) & ((0.3,0.2),(0.8,0.1)) \\ a_{2} & ((0.5,0.2), 0.9) & ((0.6,0.3), 0.6) & ((0.8,0.2),(0.6,0.1)) & ((0.7,0.2),(0.6,0.3)) \\ a_{3} & (0.7,0.1) & ((0.3,0.2),(0.7,0.2)) & ((0.7,0.1),(0.5,0.2)) & ((0.9,0.1), 0.8) \\ a_{4} & (0.8,0.2) & (0.6,(0.5,0.2)) & ((0.9,0.1), 0.5) & ((0.5,0.2),(0.7,0.1))\end{array}$

Table 3: Mixed Individual Decision Matrix $D^{(2)}$

\begin{tabular}{lllll} 
& \multicolumn{1}{c}{$c_{1}$} & $c_{2}$ & $c_{3}$ & $c_{4}$ \\
$a_{1}$ & $((0.6,0.1), 0.7)$ & $((0.6,0.2), 0.7)$ & $((0.5,0.2),(0.7,0.1))$ & $((0.5,0.2),(0.8,0.1))$ \\
$a_{2}$ & $((0.7,0.1),(0.5,0.2))$ & $((0.8,0.3),(0.6,0.2))$ & $((0.7,0.2),(0.6,0.3))$ & $((0.7,0.2),(0.6,0.3))$ \\
$a_{3}$ & $((0.5,0.3), 0.6)$ & $((0.7,0.2),(0.6,0.3))$ & $((0.7,0.1),(0.5,0.2))$ & $(0.8,0.1)$ \\
$a_{4}$ & $(0.8,0.2)$ & $((0.9,0.1), 0.8)$ & $((0.9,0.1), 0.5)$ & $((0.5,0.3),(0.8,0.1))$
\end{tabular}

Table 4: Mixed Individual Decision Matrix $D^{(3)}$

\begin{tabular}{lllll} 
& \multicolumn{1}{c}{$c_{1}$} & $c_{2}$ & $c_{3}$ & $c_{4}$ \\
$a_{1}$ & $((0.6,0.1), 0.7)$ & $((0.7,0.2),(0.6,0.3))$ & $((0.5,0.2),(0.7,0.1))$ & $((0.6,0.2), 0.9)$ \\
$a_{2}$ & $((0.6,0.1),(0.6,0.2))$ & $((0.8,0.3),(0.6,0.2))$ & $(0.7,(0.7,0.1))$ & $((0.7,0.2), 0.8)$ \\
$a_{3}$ & $(0.8,(0.7,0.1))$ & $((0.7,0.2), 0.5)$ & $((0.7,0.1),(0.5,0.2))$ & $((0.6,0.2),(0.8,0.1))$ \\
$a_{4}$ & $(0.6,(0.7,0.1))$ & $((0.9,0.1), 0.8)$ & $((0.9,0.1), 0.5)$ & $((0.5,0.3),(0.8,0.1))$
\end{tabular}

Table 5: Normalized decision matrix $\widetilde{D}$

$\begin{array}{lll} & c_{1} & c_{2} \\ a_{1} & ((0.686,0.155),(0.750,0.255)) & ((0.668,0.200),(0.605,0.255)) \\ a_{2} & ((0.605,0.132),(0.751,0.152)) & ((0.736,0.300),(0.600,0.264)) \\ a_{3} & ((0.676,0.175),(0.485,0)) & ((0.579,0.200),(0.623,0.290)) \\ a_{4} & ((0.762,0.238),(0.316,0)) & ((0.826,0.174),(0.712,0.200)) \\ & c_{3} & c_{4} \\ a_{1} & ((0.500,0.235),(0.663,0.174)) & ((0.200,0.459),(0.832,0.100)) \\ a_{2} & ((0.745,0.221),(0.510,0.147)) & ((0.200,0.700),(0.664,0.271)) \\ a_{3} & ((0.700,0.100),(0.500,0.200)) & ((0.119,0.820),(0.661,0)) \\ a_{4} & ((0.900,0.100),(0.500,0.500)) & ((0.255,0.500),(0.764,0.100))\end{array}$

Table 6: Complete Z-intuitionistic Fuzzy Number Ratio System

$\begin{array}{llll} & y_{1}\left(a_{i}\right) & S\left(y_{1}\left(a_{i}\right)\right) & s_{1}\left(a_{i}\right) \\ a_{1} & ((0.561,0.242),(0.699,0.204)) & 1.021 & 3 \\ a_{2} & ((0.628,0.289),(0.628,0.217)) & 0.994 & 4 \\ a_{3} & ((0.558,0.235),(0.569,0)) & 1.323 & 2 \\ a_{4} & ((0.765,0.217),(0.612,0)) & 1.548 & 1\end{array}$


Table 7: Complete Z-intuitionistic Fuzzy Number Reference Point

$\begin{array}{lllll} & \min _{j} \omega_{j} d\left(\tilde{Z}^{-}, \tilde{I}_{i j}\right) & \max _{j} \omega_{j} d\left(\tilde{Z I^{+}}, \tilde{Z}_{i j}\right) & y_{2}\left(a_{i}\right) & \left.s_{2}\left(a_{i}\right)\right) \\ a_{1} & 0.0298 & 0.0539 & 0.0241 & 1 \\ a_{2} & 0.0143 & 0.0511 & 0.0368 & 3 \\ a_{3} & 0.0087 & 0.0609 & 0.0522 & 4 \\ a_{4} & 0.0268 & 0.0574 & 0.0306 & 2\end{array}$

Table 8: Complete Z-intuitionistic Fuzzy Number Full Muitiplicative Form

$\begin{array}{llll} & y_{3}\left(a_{i}\right) & S\left(y_{3}\left(a_{i}\right)\right) & \left.s_{3}\left(a_{i}\right)\right) \\ a_{1} & ((0.504,0.253),(0.698,0.203)) & 0.969 & 2 \\ a_{2} & ((0.550,0.360),(0.632,0.212)) & 0.891 & 3 \\ a_{3} & ((0.461,0.384),(0.579,0.152)) & 0.854 & 4 \\ a_{4} & ((0.656,0.249),(0.569,0.212)) & 1.026 & 1\end{array}$

Table 9: Judgment matrix from DMs

\begin{tabular}{cccccccccccc}
\hline$d_{1}$ & $y_{1}$ & $y_{2}$ & $y_{3}$ & $d_{2}$ & $y_{1}$ & $y_{2}$ & $y_{3}$ & $d_{3}$ & $y_{1}$ & $y_{2}$ & $y_{3}$ \\
\hline$y_{1}$ & 1 & $1 / 8$ & $1 / 3$ & $y_{1}$ & 1 & $1 / 6$ & 2 & $y_{1}$ & 1 & 3 & 3 \\
$y_{2}$ & 8 & 1 & 6 & $y_{2}$ & 6 & 1 & 7 & $y_{2}$ & $1 / 3$ & 1 & 2 \\
$y_{3}$ & 3 & $1 / 6$ & 1 & $y_{3}$ & $1 / 2$ & $1 / 7$ & 1 & $y_{3}$ & $1 / 3$ & $1 / 2$ & 1 \\
\hline
\end{tabular}

Table 10: Z-intuitionistic Fuzzy MULTIMOORA method

$\begin{array}{cccccc} & \text { CZIFN-RS } & \text { CZIFN-RP } & \text { CZIFN-FMF } & F\left(a_{i}\right) & \text { ZIF-MULTIMOORA } \\ a_{1} & 3 & 1 & 2 & 1.598 & 1 \\ a_{2} & 4 & 3 & 3 & 3.230 & 3 \\ a_{3} & 2 & 4 & 4 & 3.539 & 4 \\ a_{4} & 1 & 2 & 1 & 1.632 & 2\end{array}$

\subsection{Comparison and discussion}

From the former introduction of CZIF-MA method, we know that the variable $k$ indicates the preference of DMs on reliability information, and different aggregation operator and distance measure are given to let CZIF-MA method be more robust and flexible. Besides, the proposed ranking aggregation tool based on AHP is robust in reflecting the DMs' preference on three subordinate rankings. Thus, to further illustrate the advantage of the proposed method, we solve the above MCGDM problem with CZIF-MA method in different distance measures, aggregation operators, the size of $k$ and different ranking aggregation tools.

\subsubsection{Sensitivity analysis}

We can get conclusions from Table 11 that diverse results can be obtained by different size of $k$, distance measure and aggregation operators, which indicate that DMs are able to 
Table 11: Ranking results with different parameters

\begin{tabular}{lllclc}
\hline & & CZIIOWA & $\begin{array}{c}\text { Optimal } \\
\text { location }\end{array}$ & CZIIOWG & $\begin{array}{c}\text { Optimal } \\
\text { location }\end{array}$ \\
\hline & $k=0$ & $a_{4} \succ a_{2} \succ a_{3} \succ a_{1}$ & $a_{4}$ & $a_{4} \succ a_{2} \succ a_{3} \succ a_{1}$ & $a_{4}$ \\
& $k=0.25$ & $a_{4} \succ a_{2} \succ a_{1} \succ a_{3}$ & $a_{4}$ & $a_{4} \succ a_{2} \succ a_{1} \succ a_{3}$ & $a_{4}$ \\
$r=1$ & $k=0.5$ & $a_{4} \succ a_{1} \succ a_{2} \succ a_{3}$ & $a_{4}$ & $a_{4} \succ a_{2} \succ a_{1} \succ a_{3}$ & $a_{4}$ \\
& $k=1$ & $a_{1} \succ a_{4} \succ a_{2} \succ a_{3}$ & $a_{1}$ & $a_{1} \succ a_{4} \succ a_{2} \succ a_{3}$ & $a_{1}$ \\
& $k=2$ & $a_{3} \succ a_{2} \succ a_{1} \succ a_{4}$ & $a_{3}$ & $a_{1} \succ a_{4} \succ a_{2} \succ a_{3}$ & $a_{1}$ \\
\hline$k=0$ & $a_{4} \succ a_{2} \succ a_{1} \succ a_{3}$ & $a_{4}$ & $a_{4} \succ a_{2} \succ a_{1} \succ a_{3}$ & $a_{4}$ \\
& $k=0.25$ & $a_{4} \succ a_{2} \succ a_{1} \succ a_{3}$ & $a_{4}$ & $a_{4} \succ a_{2} \succ a_{1} \succ a_{3}$ & $a_{4}$ \\
$r=2$ & $k=0.5$ & $a_{4} \succ a_{2} \succ a_{1} \succ a_{3}$ & $a_{4}$ & $a_{4} \succ a_{2} \succ a_{1} \succ a_{3}$ & $a_{4}$ \\
& $k=1$ & $a_{1} \succ a_{4} \succ a_{2} \succ a_{3}$ & $a_{1}$ & $a_{1} \succ a_{4} \succ a_{2} \succ a_{3}$ & $a_{1}$ \\
& $k=2$ & $a_{3} \succ a_{2} \succ a_{1} \succ a_{4}$ & $a_{3}$ & $a_{1} \succ a_{4} \succ a_{2} \succ a_{3}$ & $a_{1}$ \\
\hline
\end{tabular}

choose suitable method for MCGDM based on their preference. Especially, when $r=1$, using CZIIOWA oprator, and $k=0$, which represents reliability has no effect on decision making, the ranking is $a_{4} \succ a_{2} \succ a_{3} \succ a_{1}$, and optimal location is $a_{4}$. However, when $r=1$, using CZIIOWA oprator, and $k=2$, which represents reliability has weak effect on decision making, we get a totally different ranking, $a_{3} \succ a_{2} \succ a_{1} \succ a_{4}$, and the optimal location is $a_{3}$. It indicates that the proposed method can concern the reliability information within MCGDM problem.

Figure 2, 3, 4, 5 show the influence of $k$ to the three models in CZIF-MA method and the final ranking of alternatives with the condition of $r=1$ and using CZIIOWG operator. As we can see in Figure 2, 3, with the increasing of $k$, the values of both $S\left(y_{1}\left(a_{i}\right)\right)$ and $S\left(y_{2}\left(a_{i}\right)\right)$ decrease monotonically, and in Figure 4, the value of $y_{3}\left(a_{i}\right)$ presents more complex change. As the change of the value of three models, the ranking, obtained by each model, has changed, too. Finally, it results in the change of final ranking, which is shown in Figure 5. So, DMs can select the size of $k$ to decide how much reliability information will affect decision making. Also, the final ranking result is the same as that of the CZIFN-RP model. This result is because the DMs have a higher preference for the RP model, which shows that our method can adequately reflect the choice of the DMs.

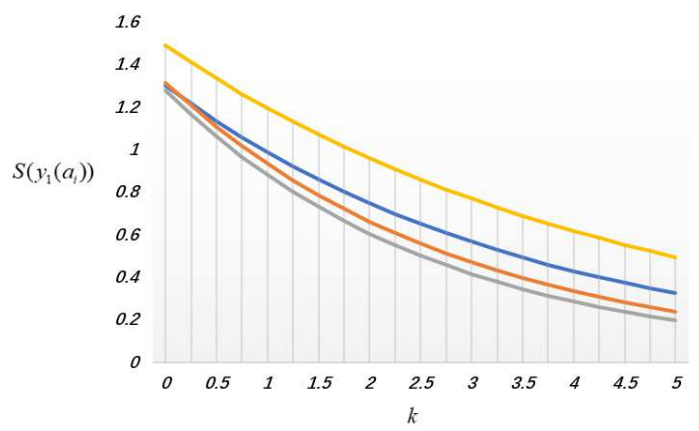

(a)

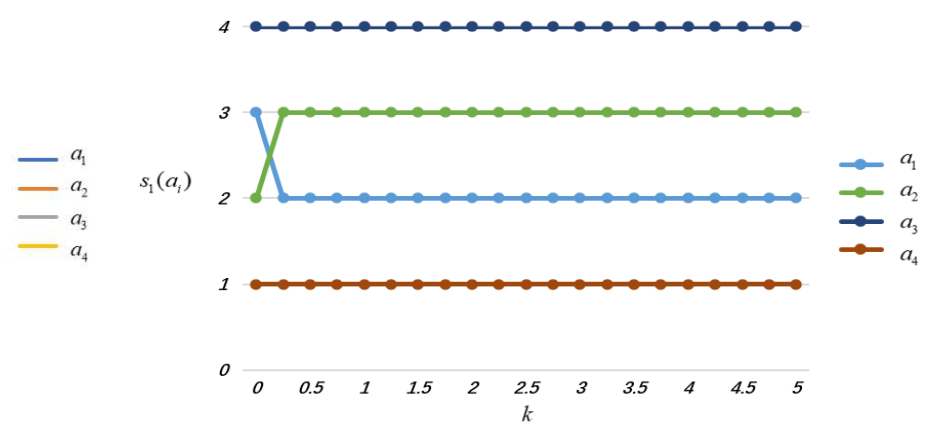

(b)

Figure 2: The influence of $k$ to the CZIFN-RS model 


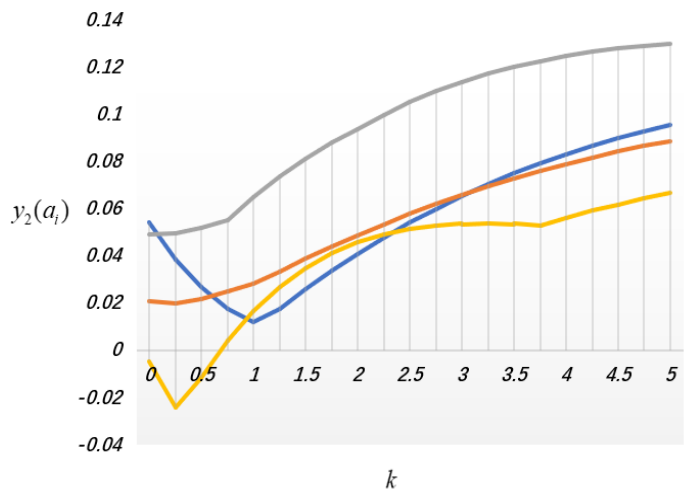

(a)

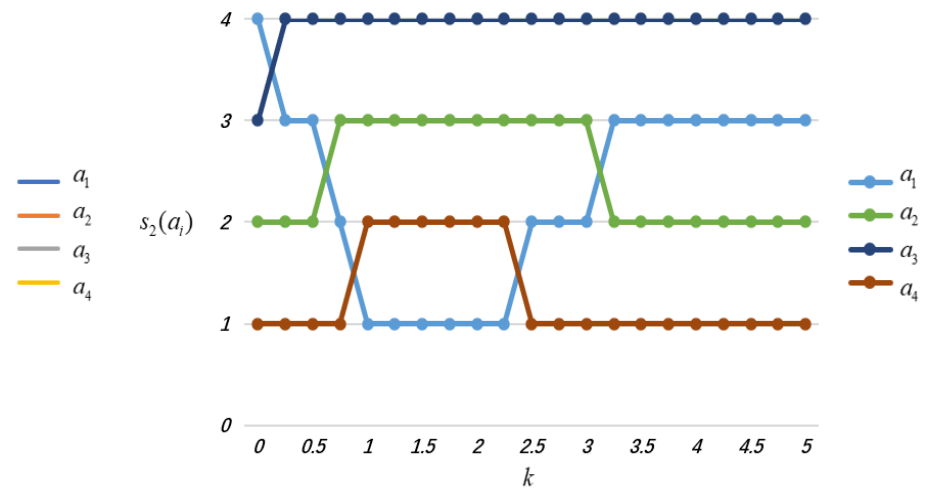

(b)

Figure 3: The influence of $k$ to the CZIFN-RP model

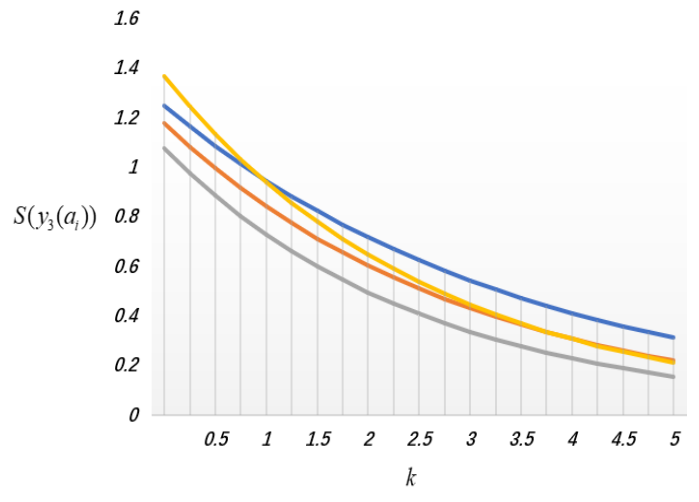

(a)

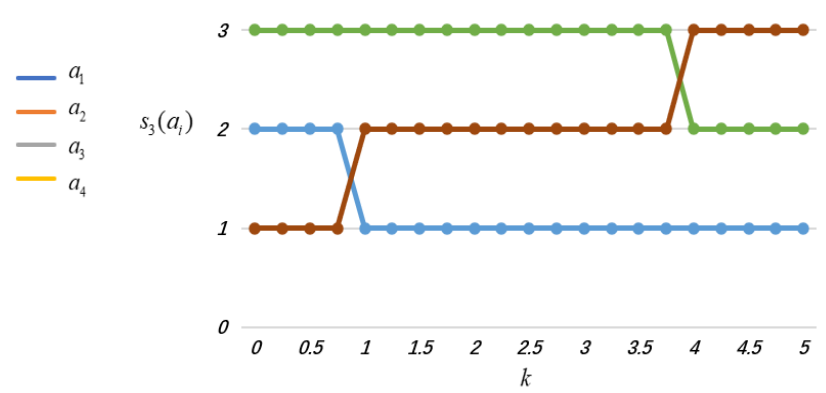

(b)

Figure 4: The influence of $k$ to the CZIFN-FMF model

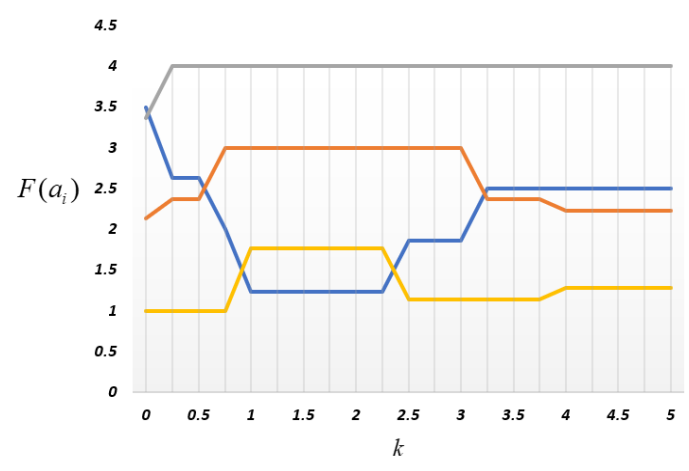

(a)

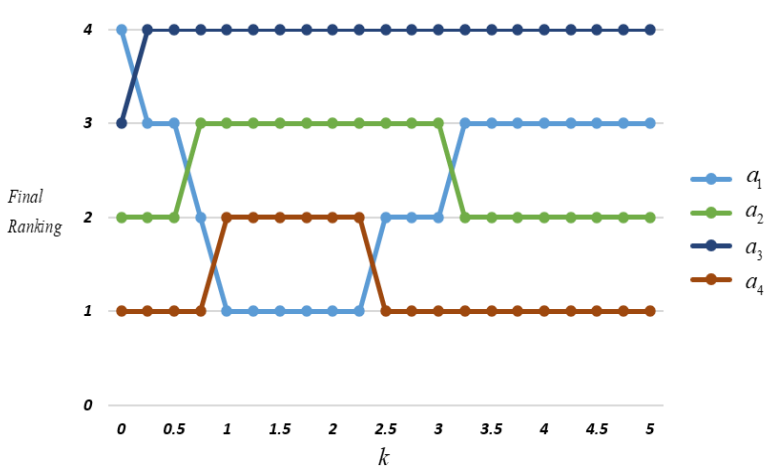

(b)

Figure 5: The influence of $k$ to the final ranking of CZIF-MA method 


\subsubsection{Comparison with different ranking aggregation tools}

All three models of MULTIMOORA method can sort and select alternertives, but their respective priorities are different. MULTIMOORA method can consider three models at the same time, making the results more reasonable. Therefore, how to consider the results of these three models simultaneously is an essential issue for scholars. The proposed ranking aggregation tool based on AHP can consider the preference of DMs on three models, when DMs aggregate three subordinate rankings into a single one. To illustrate the effectiveness of the proposed ranking aggregation tool, in Table 12, we compare the ranking results of several ranking aggregation tools with the proposed method.

As can be seen from Table 12, the optimal location obtained by previous ranking aggregation tools is all $a_{4}$, but the optimal location calculated by the proposed method is $a_{1}$ when we use the judge matrices from $d_{1}, d_{2}$ or DMs respectively, and $a_{4}$ from $d_{3}$. At the same time, the final rankings are various by different ranking aggregation tools. In proposed method, when we use the judge matrices from different DMs, it means that we consider the preference of them on three models in the process of decision-making. In Table 12, the diverse results calculated by proposed method indicate the effectiveness and superiority of proposed method to other aggregation tools while considering the preference of DMs on three models.

Table 12: Final ranking by different ranking aggregation tools

\begin{tabular}{lll}
\hline Ranking aggregation tool & Final ranking & Optimal location \\
\hline Dominance Theory[29] & $a_{4} \succ a_{1} \succ a_{2} \succ a_{3}$ & $a_{4}$ \\
Reciprocal Rank Method[44] & $a_{4} \succ a_{1} \succ a_{3} \succ a_{2}$ & $a_{4}$ \\
Improved Borda Rule[45] & $a_{4} \succ a_{3} \succ a_{1} \succ a_{2}$ & $a_{4}$ \\
Arithmetic Mean[46] & $a_{4} \succ a_{1} \succ a_{2} \succ a_{3}$ & $a_{4}$ \\
Proposed Method $\left(d_{1}\right)$ & $a_{1} \succ a_{4} \succ a_{2} \succ a_{3}$ & $a_{1}$ \\
Proposed Method $\left(d_{2}\right)$ & $a_{1} \succ a_{4} \succ a_{2} \succ a_{3}$ & $a_{1}$ \\
Proposed Method( $\left(d_{3}\right)$ & $a_{4} \succ a_{1} \succ a_{3} \succ a_{2}$ & $a_{4}$ \\
Proposed Method(DMs) & $a_{1} \succ a_{4} \succ a_{2} \succ a_{3}$ & $a_{1}$ \\
\hline
\end{tabular}

\section{Conclusion and future research}

In this study, we introduced a new class of fuzzy set, CZIFS, and its degenerate forms, AZIFS and BZIFS, which considered ambiguity information both in restraints and reliability of the information. Then, corresponding operational laws, the ranking method based on score and accuracy functions, aggregation operators of CZIFNs were proposed. Furthermore, to make full use of the new fuzzy set in the MAGDM problems, the extended MULTIMOORA method based on CZIFNs, CZIF-MA method, was given. To obtain the final ranking, the ranking aggregation tool based on AHP was integrated in CZIF-MA method. In order to solving MCGDM problem, we introduced the related decision model. Finally, the advantages of the proposed method were illustrated by a numerical example, site selecting of Fangcang shelter hospital for COVID-19.

In future research, on the one hand, we are committed to presenting effective weighting methods. On the other hand, we will work to apply our proposed model to solve more 
practical MCGDM problems such as medical diagnosis combined with other effective decision making methods.

\section{Competing interests}

The authors declare that they have no competing interests regarding the publication of this article.

\section{Acknowledgment}

This work was supported by Natural Science Foundation Project of Chongqing (Grant No.cstc2019jcyj-msxmX0716,cstc2020jcyj-msxm1397) and Science and Technology Research Program of Chongqing Municipal Education Commission (Grant No. KJQN202000601).

\section{References}

[1] C. T. Chen, Extensions of the topsis for group decision-making under fuzzy environment, Fuzzy sets and systems 114 (1) (2000) 1-9.

[2] L. Alexander Brusche, Gaps in academic literature on venture capitalists decisionmaking on funding for early-stage, high-tech ventures, Technology Transfer and Entrepreneurship 3 (2) (2016) 82-89.

[3] L. A. Zadeh, Fuzzy sets, Information and Control 8 (3) (1965) 338-353.

[4] K. Atanassov, Intuitionistic fuzzy sets, Fuzzy Sets and Systems 20 (1) (1986) 87-96.

[5] R. R. Yager, Pythagorean membership grades in multicriteria decision making, IEEE Transactions on Fuzzy Systems 22 (4) (2013) 958-965.

[6] V. Torra, Hesitant fuzzy sets, International Journal of Intelligent Systems 25 (6) (2010) 529-539.

[7] L. A. Zadeh, A note on z-numbers, Information Sciences 181 (14) (2011) 2923-2932.

[8] R. Banerjee, S. K. Pal, Z*-numbers: Augmented z-numbers for machine-subjectivity representation, Information Sciences 323 (2015) 143-178.

[9] S. D. Xian, J. H. Chai, H. L. Guo, Linguistic-induced ordered weighted averaging operator for multiple attribute group decision-making, International Journal of Intelligent Systems 34 (2) (2019) 271-296.

[10] S. Xian, Z. Yang, H. Guo, Double parameters topsis for multi-attribute linguistic group decision making based on the intuitionistic z-linguistic variables, Applied Soft Computing 85 (2019) 105835.

[11] H. G. Peng, J. Q. Wang, Hesitant uncertain linguistic z-numbers and their application in multi-criteria group decision-making problems, International Journal of Fuzzy Systems 19 (5) (2017) 1300-1316. 
[12] J. Q. Wang, Y. X. Cao, H. Y. Zhang, Multi-criteria decision-making method based on distance measure and choquet integral for linguistic z-numbers, Cognitive Computation 9 (6) (2017) 827-842.

[13] K. Khalif, K. M. Naim, A. Gegov, et al., Hybrid fuzzy mcdm model for z-numbers using intuitive vectorial centroid, Journal of Intelligent and Fuzzy Systems 33 (2) (2017) $791-805$.

[14] L. A. Gardashova, Application of operational approaches to solving decision making problem using z-numbers, Applied Mathematics 2014 (9) (2014) 1323-1334.

[15] D. Wu, X. Liu, F. Xue, et al., A new medical diagnosis method based on z-numbers, Applied Intelligence 48 (4) (2018) 854-867.

[16] B. Y. Kang, G. Chhipi Shrestha, et al., Stable strategies analysis based on the utility of z-number in the evolutionary games, Applied Mathematics and Computation 324 (2018) $202-217$.

[17] R. A. Aliev, O. H. Huseynov, R. Serdaroglu, Ranking of z-numbers and its application in decision making, International Journal of Information Technology and Decision Making 15 (06) (2016) 1503-1519.

[18] R. A. Aliev, A. V. Alizadeh, O. H. Huseynov, The arithmetic of discrete z-numbers, Information Sciences 290 (2015) 134-155.

[19] R. A. Aliev, O. H. Huseynov, L. M. Zeinalova, The arithmetic of continuous z-numbers, Information Sciences 373 (2016) 441-460.

[20] A. S. A. Bakar, A. Gegov, Multi-layer decision methodology for ranking z-numbers, International Journal of Computational Intelligence Systems 8 (2) (2015) 395-406.

[21] A. Azadeh, R. Kokabi, Z-number dea: A new possibilistic dea in the context of znumbers, Advanced engineering informatics 30 (3) (2016) 604-617.

[22] D. Qiu, Y. M. Xing, R. W. Dong, On ranking of continuous z-numbers with generalized centroids and optimization problems based on z-numbers, International Journal of Intelligent Systems 33 (1) (2018) 3-14.

[23] M. Babanli, V. Huseynov, Z-number-based alloy selection problem, Procedia Computer Science 102 (2016) 183-189.

[24] Y. Yang, J. Q. Wang, Smaa-based model for decision aiding using regret theory in discrete z-number context, Applied Soft Computing 65 (2018) 590-602.

[25] R. A. Aliev, W. Pedrycz, O. H. Huseynov, Hukuhara difference of z-numbers, Information Sciences 466 (2018) 13-24.

[26] B. Y. Kang, Y. Deng, K. o. Hewage, Generating z-number based on owa weights using maximum entropy, International Journal of Intelligent Systems 33 (8) (2018) 1745-1755. 
[27] W. Jiang, C. H. Xie, Y. Luo, Y. C. Tang, Ranking z-numbers with an improved ranking method for generalized fuzzy numbers, Journal of Intelligent and Fuzzy Systems 32 (3) (2017) 1931-1943.

[28] W. K. Brauers, E. K. Zavadskas, The moora method and its application to privatization in a transition economy, Control and cybernetics 35 (2006) 445-469.

[29] W. K. Brauers, E. K. Zavadskas, Project management by multimoora as an instrument for transition economies, Technological and Economic Development of Economy 16 (1) (2010) 5-24.

[30] P. Gupta, M. K. Mehlawat, N. Grover, et al., Multi-attribute group decision making based on extended topsis method under interval-valued intuitionistic fuzzy environment, Applied Soft Computing 69 (2018) 554-567.

[31] Z. P. Fan, X. Zhang, F. D. Chen, et al., Extended todim method for hybrid multiple attribute decision making problems, Knowledge-Based Systems 42 (2013) 40-48.

[32] H. C. Liu, L. X. Mao, Z. Y. Zhang, et al., Induced aggregation operators in the vikor method and its application in material selection, Applied Mathematical Modelling 37 (9) (2013) 6325-6338.

[33] S. Datta, N. Sahu, S. Mahapatra, Robot selection based on grey-multimoora approach, Grey Systems: Theory and Application 3 (2) (2013) 201-232.

[34] H. Souzangarzadeh, M. J. Rezvani, A. Jahan, Selection of optimum design for conical segmented aluminum tubes as energy absorbers: Application of multimoora method, Applied Mathematical Modelling 51 (2017) 546-560.

[35] X. Chen, L. Zhao, H. M. Liang, A novel multi-attribute group decision-making method based on the multimoora with linguistic evaluations, Soft Computing 22 (16) (2018) $5347-5361$.

[36] R. A. Aliev, A. V. Alizadeh, O. H. Huseynov, The arithmetic of discrete z-numbers, Information Sciences 290 (2015) 134-155.

[37] P. D. Liu, F. Jin, Methods for aggregating intuitionistic uncertain linguistic variables and their application to group decision making, Information Sciences 205 (2012) 58-71.

[38] R. R. Yager, D. P. Filev, Induced ordered weighted averaging operators, IEEE Transactions on Systems, Man, and Cybernetics, Part B (Cybernetics) 29 (2) (1999) 141-150.

[39] F. Chiclana, E. Herrera-Viedma, F. Herrera, S. Alonso, Induced ordered weighted geometric operators and their use in the aggregation of multiplicative preference relations, International Journal of Intelligent Systems 19 (3) (2004) 233-255.

[40] P. Zhao, On minkowski inequalities, Journal of Ningxia Institute of Technology(Natural Science) 03 (1995) 63-65. 
[41] H. C. Liao, R. Qin, C. Y. Gao, et al., Score-hedlisf: A score function of hesitant fuzzy linguistic term set based on hesitant degrees and linguistic scale functions: An application to unbalanced hesitant fuzzy linguistic multimoora, Information Fusion 48 (2019) $39-54$.

[42] T. L. Saaty, Axiomatic foundation of the analytic hierarchy process, Management science 32 (7) (1986) 841-855.

[43] Z. Yan, I. Lim, Y. Ge, Fangcang Shelter Hospitals for Covid-19: Construction and Operation Manual, World Scientific Publishing Company Incorporated, Singapore, 2020.

[44] S. Altuntas, T. Dereli, M. K. Yilmaz, Evaluation of excavator technologies: application of data fusion based multimoora methods, Journal of Civil Engineering and Management 21 (8) (2015) 977-997.

[45] X. L. Wu, H. C. Liao, Z. S. Xu, et al., Probabilistic linguistic multimoora: A multicriteria decision making method based on the probabilistic linguistic expectation function and the improved borda rule, IEEE Transactions on Fuzzy Systems 26 (6) (2018) 3688-3702.

[46] C. Karaca, A. Ulutaş, Supplier performance evaluation by using swara and multimoora, Economics management and econometrics 7 (2017) 137-151. 